\title{
How to maximize birefringence and nonlinearity of $\pi$-conjugated cyanurates
}

Jing Lu, ${ }^{\dagger, \#}$ Yu-Kun Lian, ${ }^{\dagger, \#}$ Lin Xiong, ${ }^{\ddagger}$ Qian-Ru Wu, ${ }^{\ddagger}$ Min Zhao, ${ }^{\ddagger}$ Ke-Xin Shi, ${ }^{\dagger}$ Ling Chen, ${ }^{\dagger} * *$ and Li-Ming $\mathrm{Wu}^{\ddagger} * *$

${ }^{\dagger}$ Beijing Key Laboratory of Energy Conversion and Storage Materials, College of Chemistry, Beijing Normal University, Beijing 100875, China

${ }^{\ddagger}$ Key Laboratory of Theoretical and Computational Photochemistry of Ministry of Education, College of Chemistry, Beijing Normal University, Beijing 100875, P. R. China 


\section{CONTENTS}

\section{Expe rimental section}

1) Synthesis and crystal growth.

2) Characterization (PXRD, XRD, Thermal stability, Reflectance and IR spectrum, Elemental analysis and Powder second harmonic generation (SHG))

3) Theoretical calculations.

\section{Tables and Figures}

Table S1. Crystallographic data and structural refinement parameters.

Table S2. Atomic coordinates and equivalent isotropic displacement parameters.

Table S3. Major bond lengths $(\AA)$ and angles $\left(^{\circ}\right)$.

Table S4. The calculated birefringence in Figure 2.

Table S5. The predicted birefringence by the $\Delta n-\gamma$ dependence.

Table S6. Linear and nonlinear optical properties of $\mathbf{I}-\mathbf{V}$.

Figure S1. The PXRD patterns.

Figure S2. TGA and DSC curves.

Figure S3. IR spectra.

Figure S4. Experimental band gaps.

Figure S5. Powder SHG measurements of III-V at @ 532 and 1064 nm.

Figure S6. The calculated band structures and densities of states.

Figure S7. The calculated refractive index dispersion curves.

Figure S8. Energy dispersive X-ray spectroscopy (EDS) analysis.

Figure S9. The crystal structure of $\left.\mathbf{I}\left(\mathrm{Li}_{2}\left(\mathrm{HC}_{3} \mathrm{~N}_{3} \mathrm{O}_{3}\right) \cdot 2 \mathrm{H}_{2} \mathrm{O} ; \mathrm{HCYL}\right)\right)$.

Figure S10. The crystal structure of II $\left(\mathrm{Rb}_{2}\left(\mathrm{HC}_{3} \mathrm{~N}_{3} \mathrm{O}_{3}\right)\right.$; $\left.\left.\mathrm{HCYR}\right)\right)$.

Figure S11. The crystal structure of III $\left(\mathrm{LiRb}\left(\mathrm{HC}_{3} \mathrm{~N}_{3} \mathrm{O}_{3}\right) \cdot 2 \mathrm{H}_{2} \mathrm{O} ; \mathrm{HCYLR}\right)$ and IV $\left(\mathrm{NaRb}_{0.86} \mathrm{Cs}_{0.14}\left(\mathrm{HC}_{3} \mathrm{~N}_{3} \mathrm{O}_{3}\right) \cdot 2 \mathrm{H}_{2} \mathrm{O} ; \mathrm{HCYNRC}\right)$.

Figure S12. The crystal structure of $\mathbf{V}\left(\mathrm{NaRb}_{3}\left(\mathrm{H}_{2} \mathrm{C}_{3} \mathrm{~N}_{3} \mathrm{O}_{3}\right)_{4} \cdot 3 \mathrm{H}_{2} \mathrm{O} ; \mathrm{H}_{2} \mathrm{CYNR}\right)$.

Figure S13. Coordination environment of cations in $\mathbf{I}-\mathbf{V}$

Figure S14. The calculated refractive index ( $n$ ) variations of $\mathbf{I}-\mathbf{V}$.

Figure S15. The calculated birefringence $(\Delta n)$ of $\mathbf{I}-\mathbf{V}$.

\section{References}




\section{Expermental section}

Experimental reagents. $\mathrm{LiOH}\left(98 \%\right.$, Aladdin), $\mathrm{NaOH}\left(96 \%\right.$, Aladdin), $\mathrm{RbOH} \cdot \mathrm{H}_{2} \mathrm{O}(99.8 \%$, Alfa), $\mathrm{CsOH}$ (99.99\%, Aladdin), $\mathrm{H}_{3} \mathrm{C}_{3} \mathrm{~N}_{3} \mathrm{O}_{3}$ (98\%, Aladdin). All chemicals above were used without further purification.

Synthesis of single crystal I $\left(\mathrm{Li}_{2}\left(\mathrm{HC}_{3} \mathrm{~N}_{3} \mathrm{O}_{3}\right) \cdot 2 \mathrm{H}_{2} \mathrm{O} ; \mathrm{HCYL}\right)$ and II $\left(\mathrm{Rb}_{2}\left(\mathrm{HC}_{3} \mathrm{~N}_{3} \mathrm{O}_{3}\right)\right.$; HCYR). Crystals I and II were successfully synthesized by vapor liquid diffusion method in an acetone-water solution (water and acetone were used as solvent and diffused solvent, respectively, with a volu me ratio of 1:5). Firstly, $\mathrm{MOH}(\mathrm{M}=\mathrm{Li}, \mathrm{Rb})$ and $\mathrm{H}_{3} \mathrm{C}_{3} \mathrm{~N}_{3} \mathrm{O}_{3}$ were dissolved by deionized water with a ratio of 2:1 or $3: 1$ in a beaker and stirred until the solution became transparent, and then put beaker in acetone to recrystallize. The millimeter-sized I and II crystals with prismatic-shaped were obtained after the growth of 10 days. The yields are $84 \%$ and $78.6 \%$, respectively, which proves that vapor liquid diffusion method has advantages of good quality, high yield and short growth period compared with other methods. ${ }^{\mathrm{S} 1-2}$

Synthesis of single crystals III ( $\left.\mathrm{LiRbHC}_{3} \mathrm{~N}_{3} \mathrm{O}_{3} \cdot 2 \mathrm{H}_{2} \mathrm{O} ; \mathrm{HCYLR}\right), \mathrm{IV}\left(\mathrm{NaRb}_{0.84} \mathrm{Cs}_{0.16} \mathrm{HC}_{3} \mathrm{~N}_{3} \mathrm{O}_{3} \cdot 2 \mathrm{H}_{2} \mathrm{O}\right.$; HCYNRC) and V $\left(\mathrm{NaRb}_{3}\left(\mathrm{H}_{2} \mathrm{C}_{3} \mathrm{~N}_{3} \mathrm{O}_{3}\right)_{4} \cdot \mathbf{3} \mathrm{H}_{2} \mathrm{O} ; \mathrm{H}_{2} \mathrm{CYNR}\right)$. Crystals III and IV were also successfully synthesized by vapor liquid diffusion method in an acetone-water solution while $\mathbf{V}$ was obtained by slow evaporation method. The acicular III was harvested by dissolving $\mathrm{RbOH}, \mathrm{LiOH}$ and $\mathrm{H}_{3} \mathrm{C}_{3} \mathrm{~N}_{3} \mathrm{O}_{3}$ with the same molar ratio with deionized water first and then put filtrate in acetone to recrystallize; the prismatic-shaped IV was obtained by dissolving equal moles $\mathrm{RbOH}, \mathrm{CsOH}, \mathrm{NaOH}$ and $\mathrm{H}_{3} \mathrm{C}_{3} \mathrm{~N}_{3} \mathrm{O}_{3}$ with deionized water and crystallize in acetone thereafter. $\mathbf{V}$ was grown by dissolving $\mathrm{RbOH}, \mathrm{NaOH}$ and $\mathrm{H}_{3} \mathrm{C}_{3} \mathrm{~N}_{3} \mathrm{O}_{3}$ with the same molar ratio with deionized water and then put filtrate to volatilize at room temperature in a dust-free environment. The yields of III, IV and V were $76.8 \%, 74 \%$ and $81.2 \%$, respectively, after the growth of 5 days.

Single-crystal structure diffraction. Colorless $\mathbf{I}-\mathbf{V}$ crystals were collected on Bruker D8 Quest diffractometer equipped with Mo $K_{\alpha}$ radiation at $293 \mathrm{~K}$. The intensity, reduction and cell refinement were carried out on Bruker SAINT. All crystal structures were solved by direct method and refined through the full-matrix least-squares fitting on $\mathrm{F}^{2}$ with SHELX-97 software ${ }^{\mathrm{S} 3}$. The structures were verified by virtue of the ADDSYM algorithm from PLATON, and no higher symmetries were found.

Table 1 summarized the crystal parameters, crystal structure refinement results and data collection,

Table 2 listed the atomic coordinates and equivalent isotropic displacement parameters, and selected bond lengths and angles are presented in Table S3.

Powder X-ray diffraction. The powder XRD measurement of $\mathbf{I}-\mathbf{V}$ was carried out on a Bruker D8 ADVANCE diffractometer, using $\mathrm{Cu} K_{\alpha}$ radiation source $(\lambda=1.5418 \AA)$ at $298 \mathrm{~K}$ with a scan step of $0.02^{\circ}$ in the range of $2 \theta=10-70^{\circ}$, and the collection time is $0.05 \mathrm{~s}$ per step. 
Thermal analysis. All differential scanning calorimetric (DSC) and thermal gravimetric (TG) data were collected by NETZSCH STA 449 F5 thermal analyzer in nitrogen gas flow. The sample was packed in $\mathrm{Al}_{2} \mathrm{O}_{3}$ crucibles and heated from $35^{\circ} \mathrm{C}$ to $1000{ }^{\circ} \mathrm{C}$ at a rate of $10{ }^{\circ} \mathrm{C} \cdot \mathrm{min}^{-1}$.

UV-VIS-NIR diffuse reflectance and IR S pectrum. The diffuse spectra of $\mathbf{I}-\mathbf{V}$ were collected on a Shimadzu (Model: UV-2600) spectrophotometer in the range of 200-1000 $\mathrm{nm}$ with an integrating sphere. $\mathrm{BaSO}_{4}$ was used as a $100 \%$ reflectance reference. The absorption spectra were calculated by Kubelka-Munk function ${ }^{\mathrm{S} 4,5}: \alpha / S=(1-R)^{2} / 2 R$, where $\alpha, S$ and $R$ is the absorption coefficient, scattering coefficient and reflectance, respectively. The IR spectra were recorded on a NEXUS 670 FT-IR spectrometer (OSIC HOLDINGS) in the range of $400-4000 \mathrm{~cm}^{-1}$ on $1-2 \mathrm{mg}$ powder samples mixed thoroughly with about $100 \mathrm{mg} \mathrm{KBr}$.

Elemental analysis. The semiquantitative ele mental analysis spectra were performed on selected single crystal surfaces by a field emis sion scanning electron mic roscopy (FESEM, S-8010, Hitachi) equipped with an energy dispersive X-ray spectroscope (EDX).

Second har monic gener ation (S HG) measurement. Since III, IV and V all crystallize in polar space groups, SHG measurement were measured on polycrystalline samples using Q-s witched Nd:YAG laser at 532 and $1064 \mathrm{nmvia}$ Kurtz and Perry method ${ }^{\mathrm{S} 6}$. The polycrystalline sample were carefully screened into particle size of 25-45, 45-75, 75-109, 109-150 and 150-212 $\mu \mathrm{m}$, respectively, and reported $\mathrm{LiK}\left(\mathrm{HC}_{3} \mathrm{~N}_{3} \mathrm{O}_{3}\right) \cdot 2 \mathrm{H}_{2} \mathrm{O}^{16}$ and $\mathrm{Cs}_{3} \mathrm{Na}\left(\mathrm{H}_{2} \mathrm{C}_{3} \mathrm{~N}_{3} \mathrm{O}_{3}\right)_{4} \cdot 3 \mathrm{H}_{2} \mathrm{O}^{18}$ were also synthesized to test the reliability and reproducibility of our measurements. Commercial BBO and KDP were sieved into the corresponding particle size ranges as references in 532 and $1064 \mathrm{~nm}$, respectively.

Theoretical calculations. The first-principles calculations on compounds $\mathbf{I}-\mathbf{V}$ were performed with the aid of density functional theory by the pseudopotential methods implemented in the VASP package. The total energy calculations including full structural optimizations were carried with the generalized gradient approximation (GGA) method and PBE functional. ${ }^{\mathrm{S} 7}$ The following pseudopotentials were used to simulate ion-electron interactions for all constituent elements: $\mathrm{Li} 2 \mathrm{~s}^{1}, \mathrm{Na} 2 \mathrm{p}^{6} 3 \mathrm{~s}^{1}, \mathrm{Rb} 4 \mathrm{p}^{6} 3 \mathrm{~d}^{10} 5 \mathrm{~s}^{1}$, Cs $5 p^{6} 4 d^{10} 6 s^{1}, H 1 s^{1}, C 2 s^{2} 2 p^{2}, N 2 s^{2} 2 p^{3}, O 2 s^{2} 2 p^{4}$. A kinetic energy cutoff of $500 \mathrm{eV}$ was chosen with Monkhorst-Pack $k$-point meshes spanning less than $0.02 / \AA^{3}$ in the Brillouin zone. The choice of these computational parameters are good enough to ensure the accuracy of present purpose. Based on the calculated band structures, the imag inary part of the dielectric function was calculated and the real part of the dielectric function was determined using the Kramers-Kronig transformation, and then the refractive indices $n$ and the birefringence $\Delta n$ were obtained. And SHG coefficient $d_{\mathrm{ij}}$ is calculated and listed in Table S6. 
Table S1. Crystallographic data and structural refinement parameters for $\mathbf{I}-\mathbf{V}$.

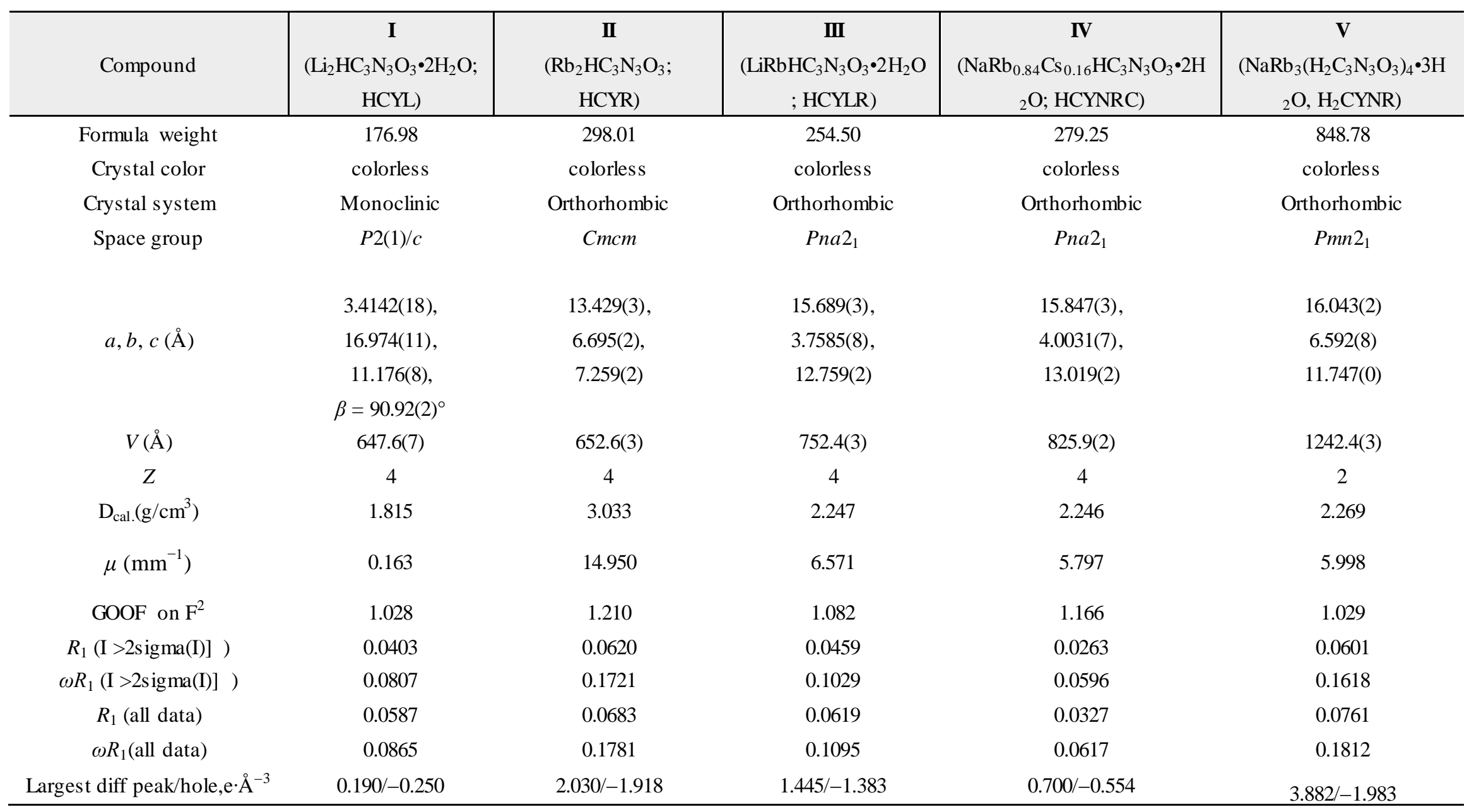


Table S2. Atomic coordinates and equivalent isotropic displacement parameters for $\mathbf{I}-\mathbf{V}$.

\begin{tabular}{|c|c|c|c|c|c|c|}
\hline \multicolumn{7}{|c|}{$\mathrm{I}\left(\mathrm{Li}_{2} \mathrm{HC}_{3} \mathrm{~N}_{3} \mathrm{O}_{3} \cdot 2 \mathrm{H}_{2} \mathrm{O} ; \mathrm{HCYL}\right)$} \\
\hline Atoms & Wyck. & Site & $x / a$ & $y / b$ & $z / c$ & $U_{\text {eq }}\left(\AA^{2}\right)^{*}$ \\
\hline $\mathrm{C}(1)$ & $4 e$ & 1.0 & $0.3654(4)$ & $0.5022(1)$ & $0.8151(1)$ & $0.017(1)$ \\
\hline $\mathrm{C}(2)$ & $4 e$ & 1.0 & $0.1355(4)$ & $0.6350(1)$ & $0.8312(1)$ & $0.016(1)$ \\
\hline$C(3)$ & $4 e$ & 1.0 & $0.2053(4)$ & $0.5756(1)$ & $0.6479(1)$ & $0.016(1)$ \\
\hline $\mathrm{Li}(1)$ & $4 e$ & 1.0 & $0.6897(8)$ & $0.5728(2)$ & $0.4426(2)$ & $0.028(1)$ \\
\hline $\mathrm{Li}(2)$ & $4 e$ & 1.0 & $0.4767(8)$ & $0.3387(1)$ & $0.8070(2)$ & $0.026(1)$ \\
\hline $\mathrm{N}(1)$ & $4 e$ & 1.0 & $0.2735(4)$ & $0.5672(1)$ & $0.8824(1)$ & $0.018(1)$ \\
\hline $\mathrm{N}(2)$ & $4 e$ & 1.0 & $0.1000(4)$ & $0.6396(1)$ & $0.7130(1)$ & $0.018(1)$ \\
\hline $\mathrm{N}(3)$ & $4 e$ & 1.0 & $0.3251(3)$ & $0.5062(1)$ & $0.6963(1)$ & $0.018(1)$ \\
\hline $\mathrm{O}(1)$ & $4 e$ & 1.0 & $-0.0194(3)$ & $0.2903(1)$ & $0.8615(1)$ & $0.022(1)$ \\
\hline $\mathrm{O}(2)$ & $4 e$ & 1.0 & $0.4039(4)$ & $0.3192(1)$ & $0.6345(1)$ & $0.025(1)$ \\
\hline $\mathrm{O}(3)$ & $4 e$ & 1.0 & $0.0476(3)$ & $0.6915(1)$ & $0.8990(1)$ & $0.024(1)$ \\
\hline $\mathrm{O}(4)$ & $4 e$ & 1.0 & $0.1910(3)$ & $0.5801(1)$ & $0.5343(1)$ & $0.022(1)$ \\
\hline $\mathrm{O}(5)$ & $4 e$ & 1.0 & $0.4866(3)$ & $0.4420(1)$ & $0.8690(1)$ & $0.024(1)$ \\
\hline $\mathrm{H}(1)$ & $4 e$ & 1.0 & $-0.0261(7)$ & $0.2967(1)$ & $0.937(2)$ & $0.054(7)$ \\
\hline $\mathrm{H}(2)$ & $4 e$ & 1.0 & $-0.0351(6)$ & $0.2395(1)$ & $0.8450(2)$ & $0.057(6)$ \\
\hline $\mathrm{H}(3)$ & $4 e$ & 1.0 & $0.601(9)$ & $0.3133(1)$ & $0.579(3)$ & $0.085(1)$ \\
\hline $\mathrm{H}(4)$ & $4 e$ & 1.0 & $0.248(7)$ & $0.2781(2)$ & $0.637(2)$ & $0.088(9)$ \\
\hline $\mathrm{H}(5)$ & $4 e$ & 1.0 & $0.317(5)$ & $0.5637(1)$ & $0.9599(2)$ & $0.033(5)$ \\
\hline \multicolumn{7}{|c|}{ II $\left(\mathrm{Rb}_{2} \mathrm{HC}_{3} \mathrm{~N}_{3} \mathrm{O}_{3} ; \mathrm{HCYR}\right)$} \\
\hline Atoms & Wyck. & Site & $x / a$ & $y / b$ & $z / c$ & $\operatorname{Ueq}\left(\AA^{2}\right)^{*}$ \\
\hline $\mathrm{Rb}(1)$ & $8 e$ & 1.0 & $0.8222(1)$ & 0.5000 & 0.5000 & $0.019(1)$ \\
\hline $\mathrm{O}(2)$ & $4 c$ & 1.0 & 0.5000 & $0.9415(1)$ & 0.2500 & $0.020(2)$ \\
\hline $\mathrm{O}(3)$ & $8 g$ & 1.0 & $0.6684(5)$ & $0.3536(1)$ & 0.2500 & $0.018(1)$ \\
\hline $\mathrm{N}(4)$ & $8 g$ & 1.0 & $0.5909(5)$ & $0.6573(1)$ & 0.2500 & $0.016(2)$ \\
\hline $\mathrm{N}(5)$ & $4 c$ & 1.0 & 0.5000 & $0.3602(2)$ & 0.2500 & $0.014(2)$ \\
\hline$C(6)$ & $8 g$ & 1.0 & $0.5894(6)$ & $0.4588(1)$ & 0.2500 & $0.009(2)$ \\
\hline$C(7)$ & $4 c$ & 1.0 & 0.5000 & $0.7510(2)$ & 0.2500 & $0.017(2)$ \\
\hline $\mathrm{H}(1)$ & $4 c$ & 1.0 & 0.5000 & $0.24(2)$ & 0.2500 & $0.00(3)$ \\
\hline \multicolumn{7}{|c|}{ III $\left(\mathrm{LiRbHC}_{3} \mathrm{~N}_{3} \mathrm{O}_{3} \cdot 2 \mathrm{H}_{2} \mathrm{O} ; \mathrm{HCYLR}\right)$} \\
\hline Atoms & Wyck. & Site & $x / a$ & $y / b$ & $z / c$ & $\operatorname{Ueq}\left(\AA^{2}\right):$ \\
\hline $\mathrm{N}(2)$ & $4 a$ & 1.0 & $0.4369(5)$ & $0.4930(2)$ & $0.4519(7)$ & $0.026(2)$ \\
\hline $\mathrm{O}(4)$ & $4 a$ & 1.0 & $0.3176(5)$ & $0.4806(2)$ & $0.3536(6)$ & $0.031(2)$ \\
\hline $\mathrm{Li}(1)$ & $4 a$ & 1.0 & $0.1855(2)$ & $1.4700(5)$ & $0.3302(2)$ & $0.038(4)$ \\
\hline $\mathrm{O}(2)$ & $4 a$ & 1.0 & $0.1687(6)$ & $0.9900(2)$ & $0.4009(9)$ & $0.034(3)$ \\
\hline$C(2)$ & $4 a$ & 1.0 & $0.3391(5)$ & $0.8100(3)$ & $0.6052(8)$ & $0.025(2)$ \\
\hline $\mathrm{O}(3)$ & $4 a$ & 1.0 & $0.2992(3)$ & $0.9512(1)$ & $0.6800(1)$ & $0.028(1)$ \\
\hline $\mathrm{N}(3)$ & $4 a$ & 1.0 & $0.4249(4)$ & $0.7310(2)$ & $0.6192(7)$ & $0.028(2)$ \\
\hline$C(3)$ & $4 a$ & 1.0 & $0.3529(7)$ & $0.5570(4)$ & $0.4383(1)$ & $0.023(3)$ \\
\hline $\mathrm{O}(5)$ & $4 a$ & 1.0 & $0.5510(4)$ & $0.5294(2)$ & $0.5612(6)$ & $0.032(2)$ \\
\hline
\end{tabular}


Supporting Information

\begin{tabular}{|c|c|c|c|c|c|c|}
\hline $\mathrm{Rb}(1)$ & $4 a$ & 1.0 & $0.6557(1)$ & $0.0408(2)$ & $0.6795(1)$ & $0.029(1)$ \\
\hline $\mathrm{N}(1)$ & $4 a$ & 1.0 & $0.3029(4)$ & $0.7210(2)$ & $0.5150(7)$ & $0.026(2)$ \\
\hline $\mathrm{O}(1)$ & $4 a$ & 1.0 & $0.5214(7)$ & $0.0340(2)$ & $0.3169(8)$ & $0.051(3)$ \\
\hline $\mathrm{C}(1)$ & $4 a$ & 1.0 & $0.4727(5)$ & $0.5780(2)$ & $0.5422(8)$ & $0.023(2)$ \\
\hline $\mathrm{H}(1)$ & $4 a$ & 1.0 & $0.489(1)$ & $0.17(4)$ & $0.356(1)$ & $0.06(4)$ \\
\hline $\mathrm{H}(2)$ & $4 a$ & 1.0 & $0.508(7)$ & $-0.14(3)$ & $0.335(9)$ & $0.02(3)$ \\
\hline $\mathrm{H}(4)$ & $4 a$ & 1.0 & $0.203(7)$ & $0.96(2)$ & $0.430(8)$ & $0.00(2)$ \\
\hline $\mathrm{H} 3 \mathrm{~A}$ & $4 a$ & 1.0 & $0.448(5)$ & $0.7805(0)$ & $0.678(3)$ & 0.034 \\
\hline $\mathrm{H} 3 \mathrm{~B}$ & $4 a$ & 1.0 & $0.118(1)$ & $1.010(4)$ & $0.429(1)$ & 0.034 \\
\hline \multicolumn{7}{|c|}{ IV $\left(\mathrm{NaRb}_{0.84} \mathrm{Cs}_{0.16} \mathrm{HC}_{3} \mathrm{~N}_{3} \mathrm{O}_{3} \cdot 2 \mathrm{H}_{2} \mathrm{O} ; \mathrm{HCYNRC}\right)$} \\
\hline Atoms & Wyck. & Site & $x / a$ & $y / b$ & $z / c$ & $\operatorname{Ueq}\left(\AA^{2}\right)^{*}$ \\
\hline $\mathrm{C}(1)$ & $4 a$ & 1.0 & $0.5255(3)$ & $0.4225(1)$ & $0.4948(4)$ & $0.024(1)$ \\
\hline$C(2)$ & $4 a$ & 1.0 & $0.6606(3)$ & $0.1927(1)$ & $0.5432(4)$ & $0.025(1)$ \\
\hline $\mathrm{C}(3)$ & $4 a$ & 1.0 & $0.6399(4)$ & $0.4329(2)$ & $0.3823(6)$ & $0.023(1)$ \\
\hline $\mathrm{N}(1)$ & $4 a$ & 1.0 & $0.5773(3)$ & $0.2664(1)$ & $0.5643(4)$ & $0.028(1)$ \\
\hline $\mathrm{N}(2)$ & $4 a$ & 1.0 & $0.6919(2)$ & $0.2812(1)$ & $0.4525(4)$ & $0.025(1)$ \\
\hline $\mathrm{N}(3)$ & $4 a$ & 1.0 & $0.5568(3)$ & $0.5020(1)$ & $0.4020(3)$ & $0.024(1)$ \\
\hline $\mathrm{Na}(1)$ & $4 a$ & 1.0 & $0.6786(2)$ & $0.0049(5)$ & $0.7837(2)$ & $0.030(1)$ \\
\hline $\mathrm{O}(1)$ & $4 a$ & 1.0 & $0.5215(3)$ & $-0.0341(1)$ & $0.7695(3)$ & $0.033(1)$ \\
\hline $\mathrm{O}(2)$ & $4 a$ & 1.0 & $0.6676(3)$ & $0.4945(1)$ & $0.8706(5)$ & $0.032(1)$ \\
\hline $\mathrm{O}(3)$ & $4 a$ & 1.0 & $0.7033(2)$ & $0.0513(1)$ & $0.6118(3)$ & $0.034(1)$ \\
\hline $\mathrm{O}(4)$ & $4 a$ & 1.0 & $0.4507(2)$ & $0.4763(9)$ & $0.5203(3)$ & $0.035(1)$ \\
\hline $\mathrm{O}(5)$ & $4 a$ & 1.0 & $0.6696(3)$ & $0.5052(9)$ & $0.2961(3)$ & $0.030(1)$ \\
\hline $\mathrm{Rb}(1)$ & $4 a$ & 0.86 & $0.6538(1)$ & $0.0300(1)$ & $0.1288(1)$ & $0.029(1)$ \\
\hline $\operatorname{Cs}(1)$ & $4 a$ & 0.14 & $0.6538(1)$ & $0.0300(1)$ & $0.1288(1)$ & $0.029(1)$ \\
\hline $\mathrm{H}(1)$ & $4 a$ & 1.0 & $0.713(2)$ & $0.545(1)$ & $0.902(5)$ & $0.029(1)$ \\
\hline $\mathrm{H}(2)$ & $4 a$ & 1.0 & $0.624(5)$ & $0.49(2)$ & $0.909(8)$ & $0.08(4)$ \\
\hline $\mathrm{H}(3)$ & $4 a$ & 1.0 & $0.499(4)$ & $-0.146(1)$ & $0.790(5)$ & $0.04(2)$ \\
\hline $\mathrm{H}(4)$ & $4 a$ & 1.0 & $0.497(6)$ & $0.172(2)$ & $0.809(6)$ & $0.09(3)$ \\
\hline $\mathrm{H}(5)$ & $4 a$ & 1.0 & $0.560(3)$ & $0.203(1)$ & $0.623(2)$ & $0.048(1)$ \\
\hline \multicolumn{7}{|c|}{$\mathrm{V}\left(\mathrm{NaRb}_{3}\left(\mathrm{H}_{2} \mathrm{C}_{3} \mathrm{~N}_{3} \mathrm{O}_{3}\right)_{4} \bullet \mathbf{3} \mathrm{H}_{2} \mathrm{O} ; \mathrm{H}_{2} \mathrm{CYNR}\right)$} \\
\hline Atoms & Wyck. & Site & $x / a$ & $y / b$ & $z / c$ & $\operatorname{Ueq}\left(\AA^{2}\right)^{*}$ \\
\hline $\mathrm{Na}(1)$ & $2 a$ & $m$ & 0.5000 & $0.2744(1)$ & $0.5423(9)$ & $0.038(2)$ \\
\hline $\mathrm{O}(1)$ & $2 a$ & $m$ & 0.5000 & $0.3158(8)$ & $0.8347(3)$ & $0.033(3)$ \\
\hline $\mathrm{N}(1)$ & $4 b$ & 1.0 & $0.3569(9)$ & $0.195(2)$ & $0.4506(1)$ & $0.032(3)$ \\
\hline $\mathrm{C}(1)$ & $4 b$ & 1.0 & $0.8129(9)$ & $0.5080(2)$ & $0.7176(1)$ & $0.024(3)$ \\
\hline $\mathrm{Rb} 1 \mathrm{~A}$ & $2 a$ & $m$ & 0.10000 & $0.2525(6)$ & $0.3533(8)$ & $0.108(2)$ \\
\hline $\mathrm{Rb}(2)$ & $2 a$ & $m$ & 0.5000 & $0.6900(3)$ & $0.4015(6)$ & $0.034(1)$ \\
\hline $\mathrm{O}(2)$ & $2 a$ & $m$ & 0.5000 & $0.7773(6)$ & $0.1328(6)$ & $0.035(3)$ \\
\hline $\mathrm{N}(2)$ & $4 b$ & 1.0 & $0.2307(1)$ & $-0.013(10)$ & $0.5542(1)$ & $0.029(3)$ \\
\hline $\mathrm{C}(2)$ & $4 b$ & 1.0 & $0.8177(7)$ & $0.4760(20)$ & $0.5226(1)$ & $0.019(3)$ \\
\hline $\mathrm{O}(4)$ & $4 b$ & 1.0 & $0.8494(8)$ & $0.5141(2)$ & $0.8100(1)$ & $0.039(3)$ \\
\hline $\mathrm{N}(4)$ & $4 b$ & 1.0 & $0.7286(1)$ & $0.5015(2)$ & $0.5215(1)$ & $0.021(2)$ \\
\hline $\mathrm{C}(4)$ & $4 b$ & 1.0 & $0.3107(1)$ & $0.0330(2)$ & $0.5517(1)$ & $0.031(4)$ \\
\hline
\end{tabular}


Supporting Information

\begin{tabular}{ccccccc}
\hline $\mathrm{C}(3)$ & $4 b$ & 1.0 & $0.6858(8)$ & $0.5340(2)$ & $0.6208(1)$ & $0.024(3)$ \\
$\mathrm{Rb}(3)$ & $2 a$ & $m$ & 0.5000 & $0.1910(3)$ & $0.1653(6)$ & $0.031(1)$ \\
$\mathrm{O}(3)$ & $4 b$ & 1.0 & $0.6060(6)$ & $0.5576(1)$ & $0.6173(1)$ & $0.033(3)$ \\
$\mathrm{N}(3)$ & $4 b$ & 1.0 & $0.2290(1)$ & $-0.0373(8)$ & $0.3561(1)$ & $0.030(3)$ \\
$\mathrm{C}(5)$ & $4 b$ & 1.0 & $0.3161(9)$ & $-0.0100(2)$ & $0.3547(1)$ & $0.022(3)$ \\
$\mathrm{O}(5)$ & $4 b$ & 1.0 & $0.8493(7)$ & $0.4377(1)$ & $0.4302(1)$ & $0.034(3)$ \\
$\mathrm{N}(5)$ & $4 b$ & 1.0 & $0.7297(1)$ & $0.5370(6)$ & $0.7121(1)$ & $0.025(2)$ \\
$\mathrm{O}(6)$ & $4 b$ & $m$ & 0.5000 & $0.2447(8)$ & $0.4365(3)$ & $0.045(5)$ \\
$\mathrm{N}(6)$ & $4 b$ & 1.0 & $0.8570(7)$ & $0.4805(1)$ & $0.6166(1)$ & $0.021(2)$ \\
$\mathrm{C}(6)$ & $4 b$ & 1.0 & $0.1822(1)$ & $-0.0350(2)$ & $0.4596(1)$ & $0.023(3)$ \\
$\mathrm{O}(7)$ & $4 b$ & 1.0 & $0.3508(7)$ & $-0.0086(1)$ & $0.2605(9)$ & $0.031(3)$ \\
$\mathrm{O}(8)$ & $4 b$ & 1.0 & $0.3499(7)$ & $0.0710(1)$ & $0.6463(1)$ & $0.037(3)$ \\
$\mathrm{O}(9)$ & $4 b$ & 1.0 & $0.1101(7)$ & $-0.0596(1)$ & $0.4603(1)$ & $0.034(2)$ \\
$\mathrm{H}(1)$ & $4 b$ & 1.0 & $0.18611(4)$ & $-0.0107(1)$ & $0.61339(6)$ & $0.04(4)$ \\
$\mathrm{H}(2)$ & $4 b$ & 1.0 & $0.20899(9)$ & $-0.0483(1)$ & $0.28488(5)$ & 0.0510 \\
$\mathrm{H}(3)$ & $4 b$ & 1.0 & $0.6938(5)$ & $0.5209(7)$ & $0.77026(6)$ & $0.04(5)$ \\
$\mathrm{H}(4)$ & $4 b$ & 1.0 & $0.70693(8)$ & $0.504(5)$ & $0.45088(5)$ & 0.0500 \\
$\mathrm{H}(5)$ & $4 b$ & $m$ & $0.5349(4)$ & $0.6808(1)$ & $0.128(6)$ & 0.0510 \\
$\mathrm{H}(6)$ & $2 a$ & $m$ & 0.5 & $0.8800(9)$ & $0.0890(7)$ & $0.02(5)$ \\
$\mathrm{H}(7)$ & $2 a$ & $m$ & 0.5 & $0.1532(1)$ & $0.4875(5)$ & 0.0460 \\
$\mathrm{H}(8)$ & $2 a$ & $m$ & 0.5 & $0.1925(1)$ & $0.3704(4)$ & 0.0560 \\
$\mathrm{H}(9)$ & $2 a$ & $m$ & 0.5 & $0.277(2)$ & $0.9036(4)$ & 0.0520 \\
$\mathrm{H}(10)$ & $2 a$ & $m$ & 0.5 & $0.4173(9)$ & $0.7902(6)$ & 0.0520 \\
\hline & 4 & $m$ & & &
\end{tabular}

$* U(\mathrm{eq})$ is defined as one third of the trace of the orthogonalized $U_{\mathrm{ij}}$ tensor.

Table S3. Selected bond lengths ( $\AA$ ) and angles (deg.) for $\mathbf{I}-\mathbf{V}$

\begin{tabular}{llll}
\hline $\mathbf{I}\left(\mathbf{L i}_{2} \mathbf{H C}_{3} \mathbf{N}_{\mathbf{3}} \mathbf{O}_{3} \bullet \mathbf{2} \mathbf{H}_{2} \mathbf{O} ; \mathbf{H C Y L}\right)$ & & \\
$\mathrm{C}(1)-\mathrm{O}(5)$ & $1.2541(2)$ & $\mathrm{Li}(2)-\mathrm{O}(2)$ & $1.967(3)$ \\
$\mathrm{C}(1)-\mathrm{N}(3)$ & $1.333(2)$ & $\mathrm{Li}(2)-\mathrm{O}(1)$ & $1.988(3)$ \\
$\mathrm{C}(1)-\mathrm{N}(1)$ & $1.3740(2)$ & $\mathrm{Li}(2)-\mathrm{O}(1)$ & $1.994(3)$ \\
$\mathrm{C}(2)-\mathrm{O}(3)$ & $1.2617(2)$ & $\mathrm{O}(2)-\mathrm{H}(4)$ & $0.88(3)$ \\
$\mathrm{C}(2)-\mathrm{N}(2)$ & $1.327(2)$ & $\mathrm{N}(1)-\mathrm{H}(5)$ & $0.88(2)$ \\
$\mathrm{C}(2)-\mathrm{N}(1)$ & $1.3655(2)$ & $\mathrm{O}(1)-\mathrm{H}(1)$ & $0.85(2)$ \\
$\mathrm{C}(3)-\mathrm{O}(4)$ & $1.2723(2)$ & $\mathrm{O}(1)-\mathrm{H}(2)$ & $0.88(3)$ \\
$\mathrm{C}(3)-\mathrm{N}(3)$ & $1.3569(2)$ & $\mathrm{O}(2)-\mathrm{H}(3)$ & $0.79(3)$ \\
$\mathrm{C}(3)-\mathrm{N}(2)$ & $1.3581(2)$ & $\mathrm{O}(5)-\mathrm{C}(1)-\mathrm{N}(3)$ & $123.19(1)$ \\
$\mathrm{Li}(1)-\mathrm{O}(4)$ & $1.984(3)$ & $\mathrm{O}(5)-\mathrm{C}(1)-\mathrm{N}(1)$ & $117.91(1)$ \\
$\mathrm{Li}(1)-\mathrm{O}(4)$ & $2.005(3)$ & $\mathrm{N}(3)-\mathrm{C}(1)-\mathrm{N}(1)$ & $118.90(1)$ \\
$\mathrm{Li}(1)-\mathrm{O}(2)$ & $2.048(3)$ & $\mathrm{O}(3)-\mathrm{C}(2)-\mathrm{N}(2)$ & $122.33(1)$ \\
$\mathrm{Li}(1)-\mathrm{N}(3)$ & $2.051(3)$ & $\mathrm{O}(3)-\mathrm{C}(2)-\mathrm{N}(1)$ & $118.22(1)$ \\
$\mathrm{Li}(1)-\mathrm{O}(4)$ & $2.639(3)$ & $\mathrm{N}(2)-\mathrm{C}(2)-\mathrm{N}(1)$ & $119.45(1)$ \\
$\mathrm{Li}(2)-\mathrm{O}(5)$ & $1.884(3)$ & $\mathrm{O}(4)-\mathrm{C}(3)-\mathrm{N}(3)$ & $117.14(1)$ \\
$\mathrm{O}(4)-\mathrm{C}(3)-\mathrm{N}(2)$ & $118.77(1)$ & $\mathrm{C}(2)-\mathrm{N}(2)-\mathrm{C}(3)$ & $117.78(1)$ \\
\hline
\end{tabular}


Supporting Information

\begin{tabular}{|c|c|c|c|}
\hline $\mathrm{C}(2)-\mathrm{N}(1)-\mathrm{C}(1)$ & 121.81(1) & $\mathrm{C}(1)-\mathrm{N}(3)-\mathrm{C}(3)$ & $117.82(1)$ \\
\hline $\mathrm{C}(2)-\mathrm{N}(1)-\mathrm{H}(5)$ & $121.4(1)$ & $\mathrm{H}(1)-\mathrm{O}(1)-\mathrm{H}(2)$ & 109.3(2) \\
\hline $\mathrm{C}(1)-\mathrm{N}(1)-\mathrm{H}(5)$ & $116.7(1)$ & $\mathrm{H}(3)-\mathrm{O}(2)-\mathrm{H}(4)$ & 113(3) \\
\hline $\mathrm{N}(3)-\mathrm{C}(3)-\mathrm{N}(2)$ & 124.09(1) & & \\
\hline \multicolumn{4}{|c|}{ II $\left(\mathrm{Rb}_{2} \mathrm{HC}_{3} \mathrm{~N}_{3} \mathrm{O}_{3} ; \mathrm{HCYR}\right)$} \\
\hline $\mathrm{O}(3)-\mathrm{C}(6)$ & $1.274(9)$ & $\mathrm{Rb}(1)-\mathrm{O}(3)$ & $2.919(5)$ \\
\hline $\mathrm{O}(2)-\mathrm{C}(7)$ & $1.277(2)$ & $\mathrm{Rb}(1)-\mathrm{O}(3)$ & $2.985(5)$ \\
\hline $\mathrm{N}(4)-\mathrm{C}(6)$ & $1.329(1)$ & $\mathrm{Rb}(1)-\mathrm{O}(2)$ & $3.0244(2)$ \\
\hline $\mathrm{N}(4)-\mathrm{C}(7)$ & $1.372(1)$ & $\mathrm{N}(5)-\mathrm{H}(2)$ & $0.78(2)$ \\
\hline $\mathrm{N}(5)-\mathrm{C}(6)$ & $1.370(9)$ & $\mathrm{C}(6)-\mathrm{N}(4)-\mathrm{C}(7)$ & 116.3(8) \\
\hline $\mathrm{N}(5)-\mathrm{C}(6)$ & $1.370(9)$ & $\mathrm{C}(6)-\mathrm{N}(5)-\mathrm{H}(2)$ & $118.8(5)$ \\
\hline $\mathrm{Rb}(1)-\mathrm{O}(3)$ & $2.919(5)$ & $\mathrm{O}(3)-\mathrm{C}(6)-\mathrm{N}(5)$ & 117.6(7) \\
\hline $\mathrm{Rb}(1)-\mathrm{O}(3)$ & $2.985(5)$ & $\mathrm{O}(2)-\mathrm{C}(7)-\mathrm{N}(4)$ & 117.1(6) \\
\hline $\mathrm{Rb}(1)-\mathrm{O}(2)$ & $3.0244(2)$ & $\mathrm{C}(6)-\mathrm{N}(5)-\mathrm{C}(6)$ & $122.4(1)$ \\
\hline $\mathrm{O}(3)-\mathrm{C}(6)-\mathrm{N}(4)$ & $122.7(7)$ & $\mathrm{N}(4)-\mathrm{C}(7)-\mathrm{N}(4)$ & 125.7(1) \\
\hline$N(4)-C(6)-N(5)$ & $119.7(8)$ & & \\
\hline \multicolumn{4}{|c|}{ III $\left(\mathrm{LiRbHC}_{3} \mathrm{~N}_{3} \mathrm{O}_{3} \cdot 2 \mathrm{H}_{2} \mathrm{O} ; \mathrm{HCYLR}\right)$} \\
\hline $\mathrm{N}(2)-\mathrm{C}(1)$ & $1.320(1)$ & $\mathrm{Rb}(1)-\mathrm{C}(2)$ & $3.303(9)$ \\
\hline $\mathrm{N}(2)-\mathrm{C}(3)$ & $1.351(1)$ & $\mathrm{O}(1)-\mathrm{H}(1)$ & $0.86(15)$ \\
\hline $\mathrm{O}(4)-\mathrm{C}(3)$ & $1.247(2)$ & $\mathrm{O}(1)-\mathrm{H}(2)$ & $0.69(10)$ \\
\hline $\mathrm{C}(2)-\mathrm{N}(3)$ & $1.390(1)$ & $\mathrm{C}(1)-\mathrm{N}(2)-\mathrm{C}(3)$ & 119.0(9) \\
\hline $\mathrm{C}(2)-\mathrm{O}(3)$ & $1.259(1)$ & $\mathrm{O}(3)-\mathrm{C}(2)-\mathrm{N}(1)$ & $123.5(8)$ \\
\hline $\mathrm{C}(2)-\mathrm{N}(1)$ & $1.326(1)$ & $\mathrm{O}(3)-\mathrm{C}(2)-\mathrm{N}(3)$ & 118.2(9) \\
\hline $\mathrm{N}(3)-\mathrm{C}(1)$ & $1.365(1)$ & $\mathrm{N}(1)-\mathrm{C}(2)-\mathrm{N}(3)$ & 118.2(8) \\
\hline $\mathrm{C}(3)-\mathrm{N}(1)$ & $1.396(2)$ & $\mathrm{C}(1)-\mathrm{N}(3)-\mathrm{C}(2)$ & $122.0(8)$ \\
\hline $\mathrm{O}(5)-\mathrm{C}(1)$ & $1.266(1)$ & $\mathrm{C}(1)-\mathrm{N}(3)-\mathrm{H}(3)$ & 119.0 \\
\hline $\mathrm{N}(3)-\mathrm{H}(3)$ & 0.8600 & $\mathrm{C}(2)-\mathrm{N}(3)-\mathrm{H}(3)$ & 119.0 \\
\hline $\mathrm{Li}(1)-\mathrm{O}(2)$ & $2.17(2)$ & $\mathrm{H}(1)-\mathrm{O}(1)-\mathrm{H}(2)$ & $93(1)$ \\
\hline $\mathrm{Li}(1)-\mathrm{O}(4)$ & $2.09(2)$ & $\mathrm{O}(5)-\mathrm{C}(1)-\mathrm{N}(2)$ & $123.0(9)$ \\
\hline $\mathrm{Li}(1)-\mathrm{O}(3)$ & $1.93(2)$ & $\mathrm{O}(5)-\mathrm{C}(1)-\mathrm{N}(3)$ & 117.2(9) \\
\hline $\mathrm{Li}(1)-\mathrm{O}(2)$ & $2.03(2)$ & $\mathrm{N}(2)-\mathrm{C}(1)-\mathrm{N}(3)$ & $119.8(8)$ \\
\hline $\mathrm{Rb}(1)-\mathrm{O}(1)$ & $3.297(1)$ & $\mathrm{C}(2)-\mathrm{N}(1)-\mathrm{C}(3)$ & 118.7(8) \\
\hline $\mathrm{Rb}(1)-\mathrm{O}(4)$ & $2.889(8)$ & $\mathrm{O}(4)-\mathrm{C}(3)-\mathrm{N}(2)$ & 120.3(1) \\
\hline $\mathrm{Rb}(1)-\mathrm{O}(3)$ & $2.913(5)$ & $\mathrm{O}(4)-\mathrm{C}(3)-\mathrm{N}(1)$ & 117.3(1) \\
\hline $\mathrm{Rb}(1)-\mathrm{O}(5)$ & $2.945(7)$ & $\mathrm{N}(2)-\mathrm{C}(3)-\mathrm{N}(1)$ & $122.4(1)$ \\
\hline $\mathrm{Rb}(1)-\mathrm{O}(3)$ & $2.951(5)$ & $\mathrm{H}(1)-\mathrm{O}(1)-\mathrm{H}(2)$ & $93(1)$ \\
\hline $\mathrm{Rb}(1)-\mathrm{O}(4)$ & $2.992(7)$ & $\mathrm{H}(4)-\mathrm{O}(2)-\mathrm{H}(5)$ & $124(1)$ \\
\hline $\mathrm{Rb}(1)-\mathrm{N}(1)$ & $3.272(8)$ & & \\
\hline \multicolumn{4}{|c|}{ IV $\left(\mathrm{NaRb}_{0.84} \mathrm{Cs}_{0.16} \mathrm{HC}_{3} \mathrm{~N}_{3} \mathrm{O}_{3} \cdot 2 \mathrm{H}_{2} \mathrm{O} ; \mathrm{HCYNRC}\right)$} \\
\hline $\mathrm{C}(1)-\mathrm{O}(4)$ & $1.251(6)$ & $\mathrm{O}(5)-\mathrm{Cs}(1)$ & $3.036(4)$ \\
\hline $\mathrm{C}(2)-\mathrm{O}(3)$ & $1.255(6)$ & $\mathrm{O}(1)-\mathrm{Cs}(1)$ & $3.337(5)$ \\
\hline $\mathrm{C}(3)-\mathrm{O}(5)$ & $1.251(9)$ & $\mathrm{O}(3)-\mathrm{Cs}(1)$ & $2.975(4)$ \\
\hline $\mathrm{C}(1)-\mathrm{N}(3)$ & $1.345(7)$ & $\mathrm{O}(3)-\mathrm{Cs}(1)$ & $3.088(4)$ \\
\hline $\mathrm{C}(2)-\mathrm{N}(2)$ & $1.329(6)$ & $\mathrm{O}(4)-\operatorname{Cs}(1)$ & $2.940(4)$ \\
\hline
\end{tabular}


Supporting Information

\begin{tabular}{|c|c|c|c|}
\hline $\mathrm{C}(3)-\mathrm{N}(2)$ & $1.372(9)$ & $\mathrm{C}(2)-\operatorname{Cs}(1)$ & $3.423(5)$ \\
\hline $\mathrm{N}(1)-\mathrm{C}(1)$ & $1.371(7)$ & $\mathrm{C}(1)-\operatorname{Cs}(1)$ & $3.795(5)$ \\
\hline $\mathrm{N}(1)-\mathrm{C}(2)$ & $1.381(6)$ & $\mathrm{N}(2)-\operatorname{Cs}(1)$ & $3.498(4)$ \\
\hline $\mathrm{N}(3)-\mathrm{C}(3)$ & $1.371(8)$ & $\mathrm{O}(2)-\mathrm{H}(1)$ & $0.852(2)$ \\
\hline $\mathrm{Na}(1)-\mathrm{O}(2)$ & $2.270(6)$ & $\mathrm{O}(2)-\mathrm{H}(2)$ & $0.852(2)$ \\
\hline $\mathrm{Na}(1)-\mathrm{O}(3)$ & $2.279(5)$ & $\mathrm{O}(1)-\mathrm{H}(3)$ & $0.849(2)$ \\
\hline $\mathrm{Na}(1)-\mathrm{O}(2)$ & $2.342(6)$ & $\mathrm{O}(1)-\mathrm{H}(4)$ & $0.849(2)$ \\
\hline $\mathrm{Na}(1)-\mathrm{O}(5)$ & $2.412(5)$ & $\mathrm{C}(2)-\mathrm{N}(2)-\mathrm{C}(3)$ & $119.1(5)$ \\
\hline $\mathrm{Na}(1)-\mathrm{O}(1)$ & $2.499(5)$ & $\mathrm{C}(1)-\mathrm{N}(1)-\mathrm{H}(5)$ & $122(4)$ \\
\hline $\mathrm{Rb}(1)-\mathrm{O}(3)$ & $3.088(4)$ & $\mathrm{C}(2)-\mathrm{N}(1)-\mathrm{H}(5)$ & $115(4)$ \\
\hline $\mathrm{Rb}(1)-\mathrm{O}(1)$ & $3.337(5)$ & $\mathrm{H}(3)-\mathrm{O}(1)-\mathrm{H}(4)$ & $99(9)$ \\
\hline $\mathrm{Rb}(1)-\mathrm{C}(2)$ & $3.423(5)$ & $\mathrm{O}(4)-\mathrm{C}(1)-\mathrm{N}(3)$ & $123.1(5)$ \\
\hline $\mathrm{Rb}(1)-\mathrm{O}(5)$ & $2.902(4)$ & $\mathrm{O}(4)-\mathrm{C}(1)-\mathrm{N}(1)$ & $118.1(5)$ \\
\hline $\mathrm{Rb}(1)-\mathrm{O}(4)$ & $2.940(4)$ & $\mathrm{N}(3)-\mathrm{C}(1)-\mathrm{N}(1)$ & $118.7(5)$ \\
\hline $\mathrm{Rb}(1)-\mathrm{O}(4)$ & $2.974(4)$ & $\mathrm{C}(1)-\mathrm{N}(3)-\mathrm{C}(3)$ & $118.3(5)$ \\
\hline $\mathrm{Rb}(1)-\mathrm{O}(3)$ & $2.975(4)$ & $\mathrm{O}(5)-\mathrm{C}(3)-\mathrm{N}(3)$ & $118.8(6)$ \\
\hline $\mathrm{Rb}(1)-\mathrm{O}(5)$ & $3.036(4)$ & $\mathrm{O}(5)-\mathrm{C}(3)-\mathrm{N}(2)$ & $118.3(6)$ \\
\hline $\mathrm{Rb}(1)-\mathrm{N}(2)$ & $3.498(4)$ & $\mathrm{N}(3)-\mathrm{C}(3)-\mathrm{N}(2)$ & $122.8(6)$ \\
\hline $\mathrm{Rb}(1)-\mathrm{C}(3)$ & $3.680(7)$ & $\mathrm{O}(3)-\mathrm{C}(2)-\mathrm{N}(2)$ & $123.5(4)$ \\
\hline $\mathrm{O}(4)-\operatorname{Cs}(1)$ & $2.974(4)$ & $\mathrm{O}(3)-\mathrm{C}(2)-\mathrm{N}(1)$ & $118.0(5)$ \\
\hline \multicolumn{4}{|c|}{$\mathrm{V}\left(\mathrm{NaRb}_{3}\left(\mathrm{H}_{2} \mathrm{C}_{3} \mathrm{~N}_{3} \mathrm{O}_{3}\right)_{4} \cdot 3 \mathrm{H}_{2} \mathrm{O} ; \mathrm{H}_{2} \mathrm{CYNR}\right)$} \\
\hline $\mathrm{C}(1)-\mathrm{O}(4)$ & $1.23(2)$ & $\mathrm{Rb}(1)-\mathrm{O}(1)$ & 2.854(7) \\
\hline $\mathrm{C}(2)-\mathrm{O}(5)$ & $1.224(2)$ & $\mathrm{Rb}(1)-\mathrm{O}(5)$ & $2.856(1)$ \\
\hline $\mathrm{C}(3)-\mathrm{O}(3)$ & $1.290(2)$ & $\mathrm{Rb}(1)-\mathrm{O}(5)$ & $2.856(1)$ \\
\hline $\mathrm{C}(4)-\mathrm{O}(8)$ & $1.30(2)$ & $\mathrm{Rb}(1)-\mathrm{O}(9)$ & $2.990(1)$ \\
\hline $\mathrm{C}(5)-\mathrm{O}(7)$ & $1.239(2)$ & $\mathrm{Rb}(1)-\mathrm{O}(9)$ & $2.990(1)$ \\
\hline $\mathrm{C}(6)-\mathrm{O}(9)$ & $1.168(2)$ & $\mathrm{Rb}(1)-\mathrm{O}(2)$ & $3.291(1)$ \\
\hline $\mathrm{C}(1)-\mathrm{N}(5)$ & $1.349(2)$ & $\mathrm{Rb}(1)-\mathrm{O}(3)$ & $3.486(1)$ \\
\hline $\mathrm{C}(1)-\mathrm{N}(6)$ & $1.395(2)$ & $\mathrm{Rb}(1)-\mathrm{O}(3)$ & $3.486(1)$ \\
\hline $\mathrm{C}(2)-\mathrm{N}(4)$ & $1.440(1)$ & $\mathrm{Rb}(2)-\mathrm{O}(4)$ & $2.968(1)$ \\
\hline $\mathrm{C}(2)-\mathrm{N}(6)$ & $1.272(2)$ & $\mathrm{Rb}(2)-\mathrm{O}(4)$ & $2.968(1)$ \\
\hline $\mathrm{C}(3)-\mathrm{N}(5)$ & $1.284(2)$ & $\mathrm{Rb}(2)-\mathrm{O}(3)$ & $3.176(1)$ \\
\hline $\mathrm{N}(1)-\mathrm{C}(4)$ & $1.403(2)$ & $\mathrm{Rb}(2)-\mathrm{O}(3)$ & $3.176(1)$ \\
\hline $\mathrm{N}(1)-\mathrm{C}(5)$ & $1.32(2)$ & $\mathrm{Rb}(2)-\mathrm{O}(2)$ & $3.210(9)$ \\
\hline $\mathrm{N}(2)-\mathrm{C}(6)$ & $1.375(2)$ & $\mathrm{Rb}(2)-\mathrm{O}(6)$ & $2.964(6)$ \\
\hline $\mathrm{N}(2)-\mathrm{C}(4)$ & $1.304(2)$ & $\mathrm{Rb}(2)-\mathrm{N}(1)$ & $3.214(1)$ \\
\hline $\mathrm{N}(3)-\mathrm{C}(5)$ & $1.409(1)$ & $\mathrm{Rb}(2)-\mathrm{N}(1)$ & $3.214(1)$ \\
\hline $\mathrm{N}(3)-\mathrm{C}(6)$ & $1.430(2)$ & $\mathrm{Rb}(2)-\mathrm{O}(7)$ & $3.526(1)$ \\
\hline $\mathrm{N}(4)-\mathrm{C}(3)$ & $1.372(2)$ & $\mathrm{Rb}(2)-\mathrm{O}(7)$ & $3.526(1)$ \\
\hline $\mathrm{N}(2)-\mathrm{H}(1)$ & $1.00000(3)$ & $\mathrm{Rb}(3)-\mathrm{O}(2)$ & $2.754(5)$ \\
\hline $\mathrm{N}(3)-\mathrm{H}(2)$ & $0.90000(4)$ & $\mathrm{Rb}(3)-\mathrm{O}(7)$ & $2.952(1)$ \\
\hline $\mathrm{O}(1)-\mathrm{H}(9)$ & $0.85000(2)$ & $\mathrm{Rb}(3)-\mathrm{O}(7)$ & $2.952(1)$ \\
\hline $\mathrm{O}(2)-\mathrm{H}(5)$ & $0.8500(2)$ & $\mathrm{Rb}(3)-\mathrm{O}(9)$ & $3.111(1)$ \\
\hline $\mathrm{Na}(1)-\mathrm{O}(1)$ & $2.278(1)$ & $\mathrm{Rb}(3)-\mathrm{O}(9)$ & $3.111(1)$ \\
\hline
\end{tabular}


Supporting Information

\begin{tabular}{|c|c|c|c|}
\hline $\mathrm{Na}(1)-\mathrm{O}(6)$ & $2.426(1)$ & $\mathrm{Rb}(3)-\mathrm{O}(6)$ & $3.207(8)$ \\
\hline $\mathrm{Na}(1)-\mathrm{O}(3)$ & $2.542(1)$ & $\mathrm{Rb}(3)-\mathrm{N}(6)$ & $3.207(1)$ \\
\hline $\mathrm{Na}(1)-\mathrm{O}(3)$ & $2.542(1)$ & $\mathrm{Rb}(3)-\mathrm{N}(6)$ & $3.207(1)$ \\
\hline $\mathrm{Na}(1)-\mathrm{O}(1)$ & $2.278(1)$ & $\mathrm{Rb}(3)-\mathrm{O}(4)$ & $3.538(1)$ \\
\hline $\mathrm{Na}(1)-\mathrm{O}(8)$ & $2.757(1)$ & $\mathrm{Rb}(3)-\mathrm{O}(4)$ & $3.538(1)$ \\
\hline $\mathrm{Na}(1)-\mathrm{O}(8)$ & $2.757(1)$ & $\mathrm{N}(5)-\mathrm{C}(3)-\mathrm{N}(4)$ & $116.0(1)$ \\
\hline $\mathrm{N}(2)-\mathrm{H}(1)$ & 1.0000 & $\mathrm{O}(7)-\mathrm{C}(5)-\mathrm{N}(1)$ & $122.7(1)$ \\
\hline $\mathrm{N}(4)-\mathrm{H}(3)$ & 0.9000 & $\mathrm{~N}(1)-\mathrm{C}(5)-\mathrm{N}(3)$ & $120.1(1)$ \\
\hline $\mathrm{N}(5)-\mathrm{H}(4)$ & 0.9000 & $\mathrm{H}(7)-\mathrm{O}(6)-\mathrm{H}(8)$ & $110.9(9)$ \\
\hline $\mathrm{N}(3)-\mathrm{H}(2)$ & 0.9000 & $\mathrm{O}(9)-\mathrm{C}(6)-\mathrm{N}(2)$ & $125.3(1)$ \\
\hline $\mathrm{O}(1)-\mathrm{H}(10)$ & 0.8500 & $\mathrm{H}(10)-\mathrm{O}(1)-\mathrm{H}(9)$ & $145.8(1)$ \\
\hline $\mathrm{O}(1)-\mathrm{H}(9)$ & 0.8500 & $\mathrm{O}(4)-\mathrm{C}(1)-\mathrm{N}(5)$ & $120.5(1)$ \\
\hline $\mathrm{O}(2)-\mathrm{H}(5)$ & 0.8500 & $\mathrm{~N}(5)-\mathrm{C}(1)-\mathrm{N}(6)$ & $118.7(1)$ \\
\hline $\mathrm{O}(2)-\mathrm{H}(6)$ & 0.8500 & $\mathrm{H}(5)-\mathrm{O}(2)-\mathrm{H}(6)$ & $124(3)$ \\
\hline $\mathrm{O}(6)-\mathrm{H}(7)$ & 0.8500 & $\mathrm{C}(4)-\mathrm{N}(2)-\mathrm{H}(1)$ & $137.0(7)$ \\
\hline $\mathrm{O}(6)-\mathrm{H}(8)$ & 0.8500 & $\mathrm{O}(5)-\mathrm{C}(2)-\mathrm{N}(4)$ & $115.3(1)$ \\
\hline $\mathrm{C}(5)-\mathrm{N}(1)-\mathrm{C}(4)$ & $118.1(1)$ & $\mathrm{C}(3)-\mathrm{N}(4)-\mathrm{C}(2)$ & $120.6(1)$ \\
\hline $\mathrm{O}(4)-\mathrm{C}(1)-\mathrm{N}(6)$ & $120.8(1)$ & $\mathrm{O}(8)-\mathrm{C}(4)-\mathrm{N}(1)$ & $118.6(2)$ \\
\hline $\mathrm{C}(4)-\mathrm{N}(2)-\mathrm{C}(6)$ & $124.5(1)$ & $\mathrm{N}(5)-\mathrm{C}(3)-\mathrm{O}(3)$ & $124.8(1)$ \\
\hline $\mathrm{O}(5)-\mathrm{C}(2)-\mathrm{N}(6)$ & $124.7(1)$ & $\mathrm{O}(3)-\mathrm{C}(3)-\mathrm{N}(4)$ & $119.2(1)$ \\
\hline $\mathrm{N}(6)-\mathrm{C}(2)-\mathrm{N}(4)$ & $119.8(1)$ & $\mathrm{N}(2)-\mathrm{C}(6)-\mathrm{N}(3)$ & 113.1(1) \\
\hline $\mathrm{O}(8)-\mathrm{C}(4)-\mathrm{N}(2)$ & $119.4(1)$ & $\mathrm{C}(5)-\mathrm{N}(3)-\mathrm{C}(6)$ & $122.0(1)$ \\
\hline $\mathrm{N}(2)-\mathrm{C}(4)-\mathrm{N}(1)$ & $121.9(1)$ & $\mathrm{O}(7)-\mathrm{C}(5)-\mathrm{N}(3)$ & $117.1(1)$ \\
\hline $\mathrm{C}(3)-\mathrm{N}(5)-\mathrm{C}(1)$ & $125.6(1)$ & $\mathrm{O}(9)-\mathrm{C}(6)-\mathrm{N}(3)$ & $121.7(1)$ \\
\hline $\mathrm{C}(2)-\mathrm{N}(6)-\mathrm{C}(1)$ & $119.4(1)$ & & \\
\hline
\end{tabular}


Table S4. The calculated birefringence in Figure 2.

\begin{tabular}{|c|c|c|c|c|c|c|c|c|c|c|c|c|c|}
\hline \multirow{2}{*}{$\begin{array}{l}\text { system } \\
\text { compound }\end{array}$} & \multicolumn{6}{|c|}{ Hydro-cyanurate (HCY) } & \multicolumn{7}{|c|}{ Dihydro-cyanurate $\left(\mathrm{H}_{2} \mathrm{CY}\right)$} \\
\hline & $\mathbf{I}$ & II & III & IV & 2 & 3 & $\mathbf{V}$ & 8 & 9 & 10 & 11 & 12 & 14 \\
\hline$\gamma\left({ }^{\circ}\right)$ & 19.68 & 0 & 26.19 & 27.19 & 0 & 24.89 & 24.61 & 34.63 & 34.69 & 2.029 & 31.39 & 31.56 & 25.76 \\
\hline$\Delta n$ & 0.345 & 0.4 & 0.259 & 0.238 & $0.35^{b}$ & $0.280^{a}$ & 0.389 & $0.256^{b}$ & $0.24^{b}$ & $0.397^{a}$ & $0.371^{b}$ & $0.362^{b}$ & $0.365^{a}$ \\
\hline
\end{tabular}

I-V: calculated in this work.

${ }^{a}$ : calculated in this work

${ }^{\boldsymbol{b}}$ : from refs $17-21, \Delta n_{\max }$ in cyanurate system are show $\mathrm{n}$ in red, is realized by compound $\mathbf{I I}$.

Table S5. The predicted birefringence by $\Delta n-\gamma$ Boltzmann function in Figure 2

\begin{tabular}{ccccccccc}
\hline system & Hydro-cyanurate & $(\mathbf{H C Y})$ & \multicolumn{5}{c}{ Dihydro-cyanurate } & $\left(\mathbf{H}_{\mathbf{2}} \mathbf{C Y}\right)$ \\
compound & $\mathbf{1}$ & $\mathbf{4}$ & $\mathbf{5}$ & $\mathbf{6}$ & $\mathbf{7}$ & $\mathbf{1 3}$ \\
$\boldsymbol{\gamma}\left({ }^{\circ}\right)$ & 22.73 & 18.21 & 42.93 & 18.38 & 16.39 & 16.82 \\
$\boldsymbol{\Delta} \boldsymbol{n}$ & 0.306 & 0.390 & 0.20 & 0.390 & 0.391 & 0.391 \\
\hline
\end{tabular}


Table S6. Linear and NLO properties of compounds $\mathbf{I}-\mathbf{V}$

\begin{tabular}{|c|c|c|c|c|c|}
\hline Compound & $E_{\mathrm{g}(\mathrm{exp} .)}(\mathrm{eV})$ & $E_{\mathrm{g}(\text { cal. })}(\mathrm{eV})$ & $d_{\mathbf{i j} \text { (cal..) }}(\mathrm{pm} / \mathbf{V})$ & $d_{\mathrm{ij}(\text { exp.) }}(\times \mathbf{K D P})$ & $\Delta n(@ 532 \mathrm{~nm})$ \\
\hline $\mathbf{I}$ & 5.05 & 4.39 & 1 & & 0.345 \\
\hline II & 4.96 & 4.44 & 1 & & 0.400 \\
\hline III & 5.13 & 4.76 & $\begin{array}{c}d_{15}=d_{31}=2.291 \\
d_{24}=d_{32}=1.723 \\
d_{33}=-5.11 \\
\sim 5 \mathrm{KDP}\end{array}$ & $\sim 2.7 \mathrm{KDP}$ & 0.259 \\
\hline IV & 5.16 & 4.74 & $\begin{array}{c}d_{15}=d_{31}=3.255 \\
d_{24}=d_{32}=1.565 \\
d_{33}=-6.694 \\
\sim 6 \mathrm{KDP}\end{array}$ & $\sim 3 \mathrm{KDP}$ & 0.238 \\
\hline $\mathbf{V}$ & 5.30 & 4.74 & $\begin{array}{c}d_{15}=0.63 \\
\sim 0.5 \mathrm{KDP}\end{array}$ & $\sim 0.4 \mathrm{KDP}$ & 0.389 \\
\hline
\end{tabular}
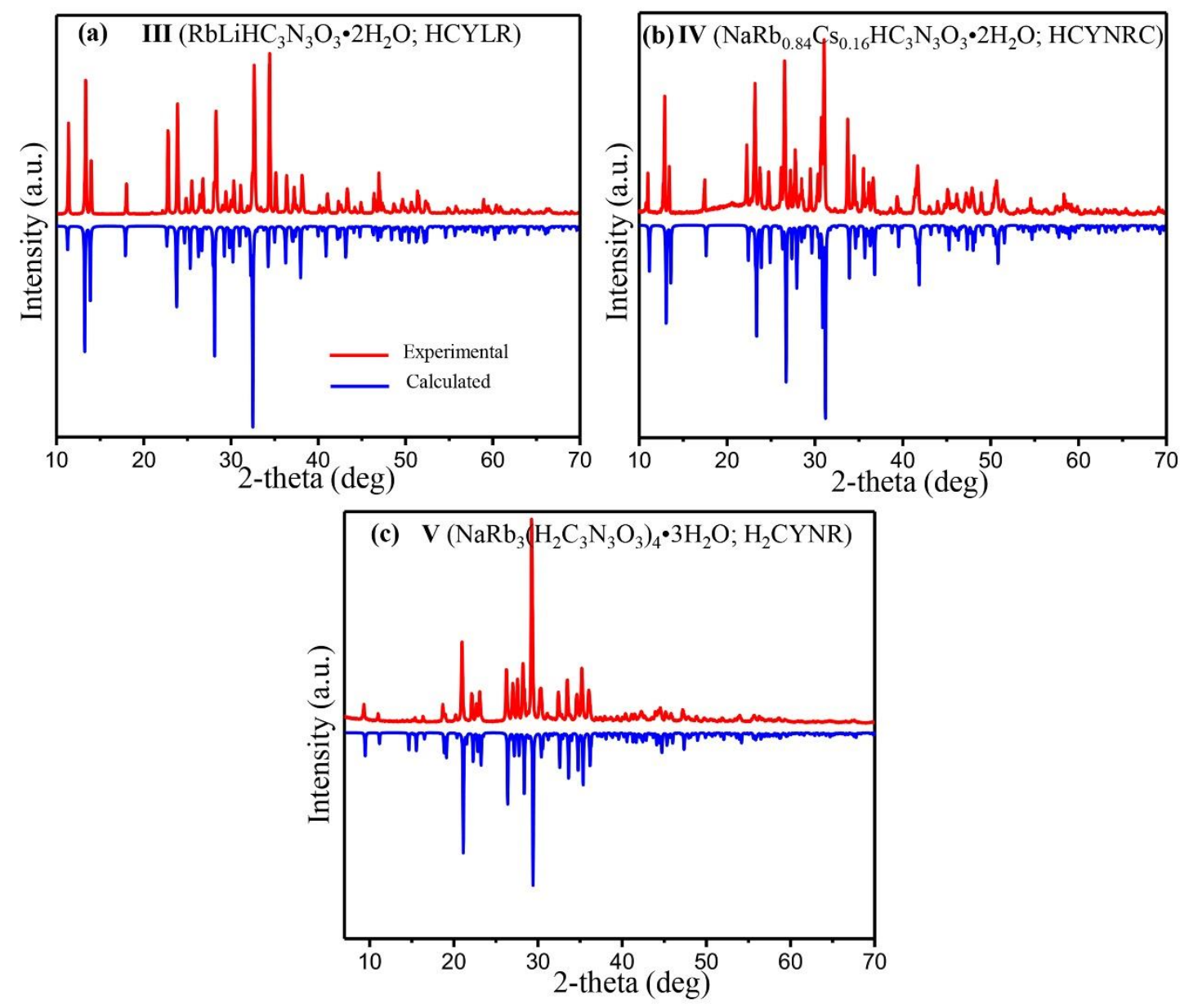

Figure S1. The PXRD patterns: I; HCYLR (a), II; HCYNRC (b) and III; $\mathrm{H}_{2}$ CYNR (c). 

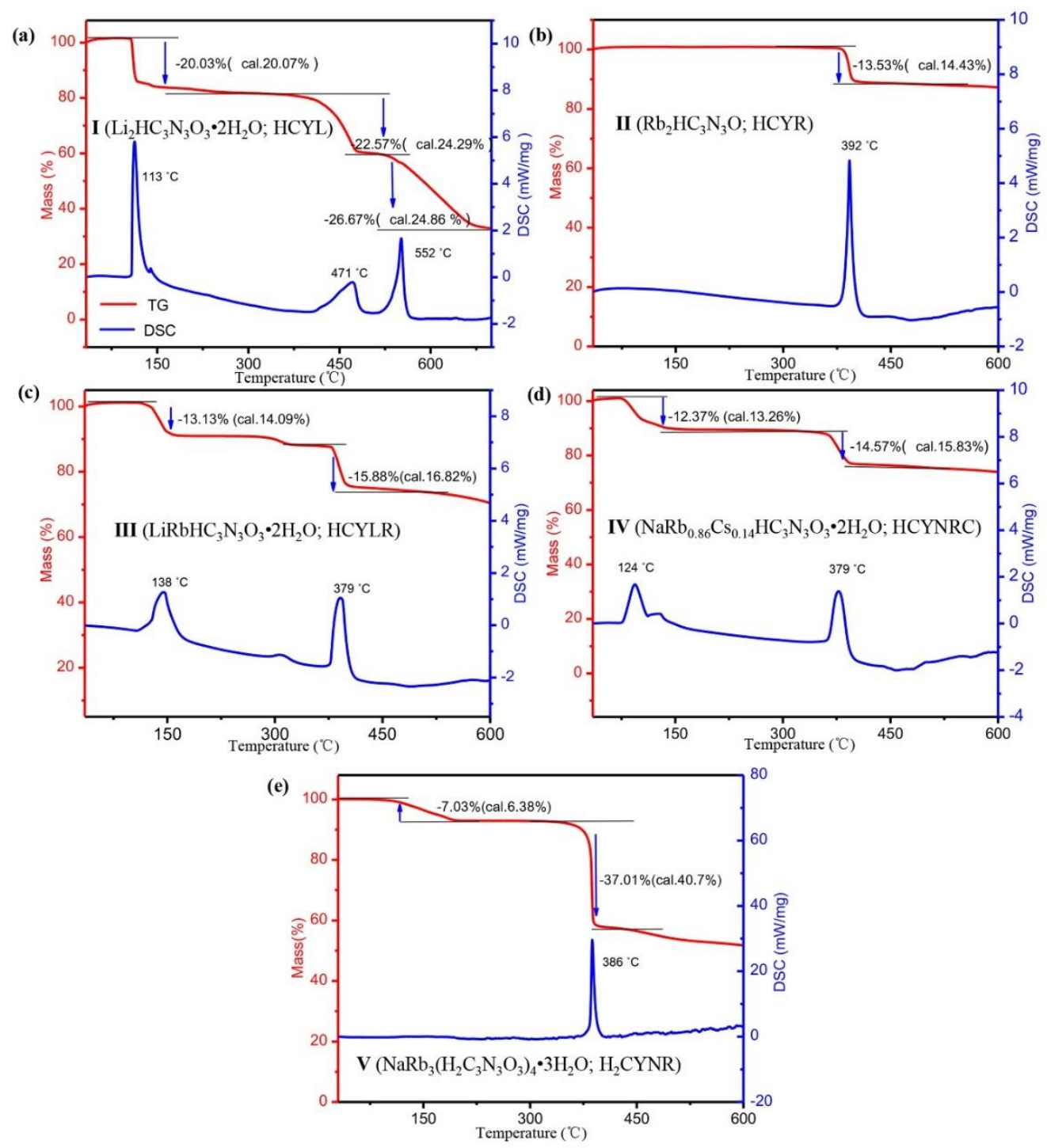

Figure S2. TGA and DSC curves: I; HCYL (a), II; HCYR (b), III; HCYLR (c), IV; HCYNRC (d) and $\mathbf{V} ; \mathrm{H}_{2} \mathrm{CYNR}$ (e). Here the decomposition process of $\mathbf{I}$; HCYL is discussed in detail as a representative. There are three main steps of mass loss as follows:

$$
\begin{aligned}
& \mathrm{Li}_{2}\left(\mathrm{HC}_{3} \mathrm{~N}_{3} \mathrm{O}_{3}\right) \cdot 2 \mathrm{H}_{2} \mathrm{O}=\mathrm{Li}_{2}\left(\mathrm{HC}_{3} \mathrm{~N}_{3} \mathrm{O}_{3}\right)+2 \mathrm{H}_{2} \mathrm{O}(\Delta m=20.34 \%) \\
& 3 \mathrm{Li}_{2}\left(\mathrm{HC}_{3} \mathrm{~N}_{3} \mathrm{O}_{3}\right)=\mathrm{Li}_{6}\left(\mathrm{C}_{3} \mathrm{~N}_{3} \mathrm{O}_{3}\right)_{2}+3 \mathrm{HNCO}(\Delta m=24.29 \%) \\
& \mathrm{Li}_{6}\left(\mathrm{C}_{3} \mathrm{~N}_{3} \mathrm{O}_{3}\right)_{2}=3 \mathrm{Li}_{2}(\mathrm{NCN})+3 \mathrm{CO}_{2} \quad(\Delta m=24.86 \%)
\end{aligned}
$$



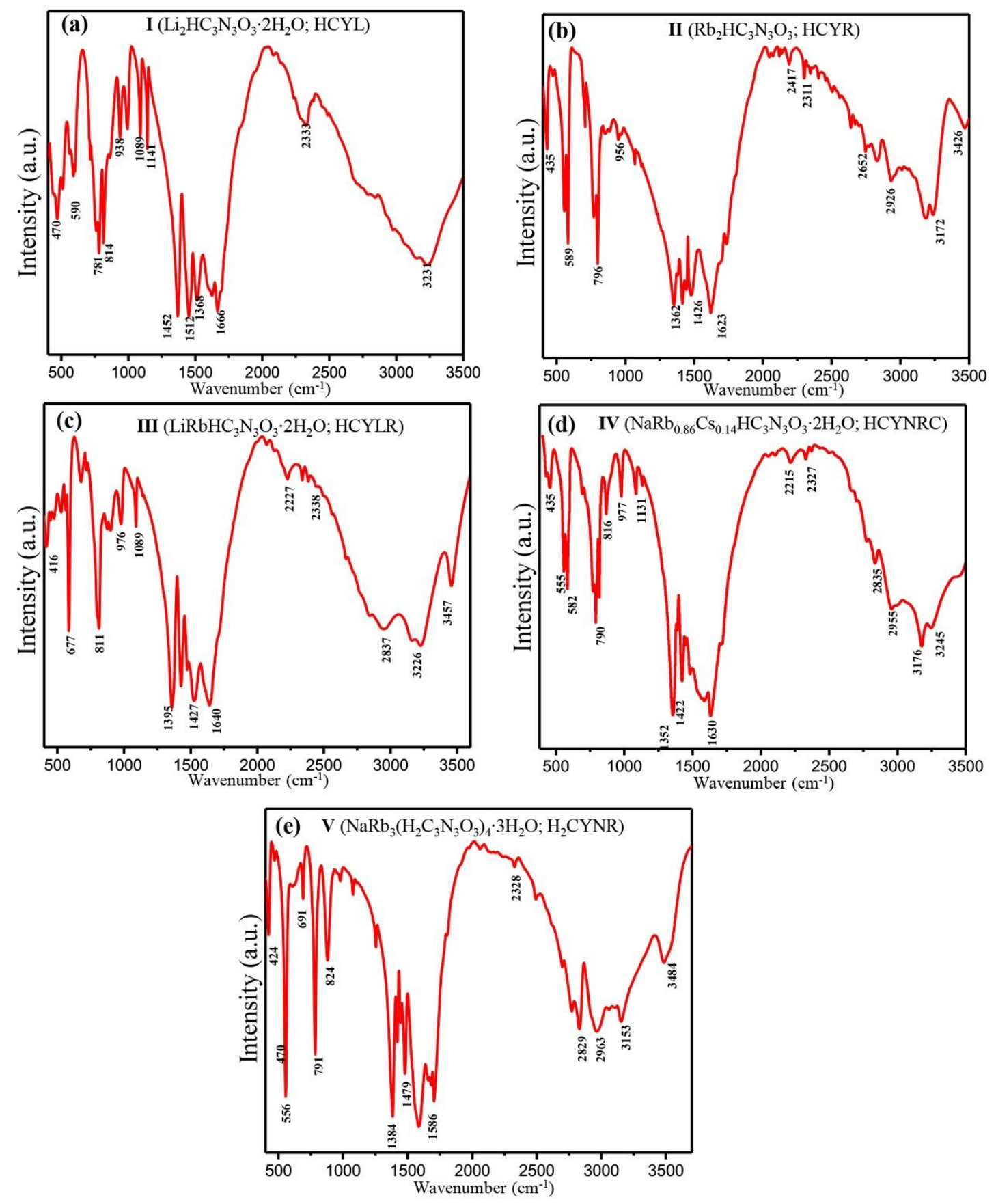

Figure S3. IR spectra of I; HCYL (a), II; HCYR (b), III; HCYLR (c), IV; HCYNRC (d) and V; $\mathrm{H}_{2}$ CYNR (e). The characteristic vibrations of CY rings in plane and out of plane were observed at 955, 814 and $584 \mathrm{~cm}^{-1}$, respectively. The corresponding $\mathrm{C}-\mathrm{O}$ bond in-plane stretching and bending vibrations were observed at 1420 and $430 \mathrm{~cm}^{-1}$, and the peaks located at 790, 1368 and $3172 \mathrm{~cm}^{-1}$ correspond to $\mathrm{N}-\mathrm{H}$ bond out-of-plane bending vibrations, in-plane bending and stretching vibrations respectively. ${ }^{\mathrm{S} 8-11}$ 

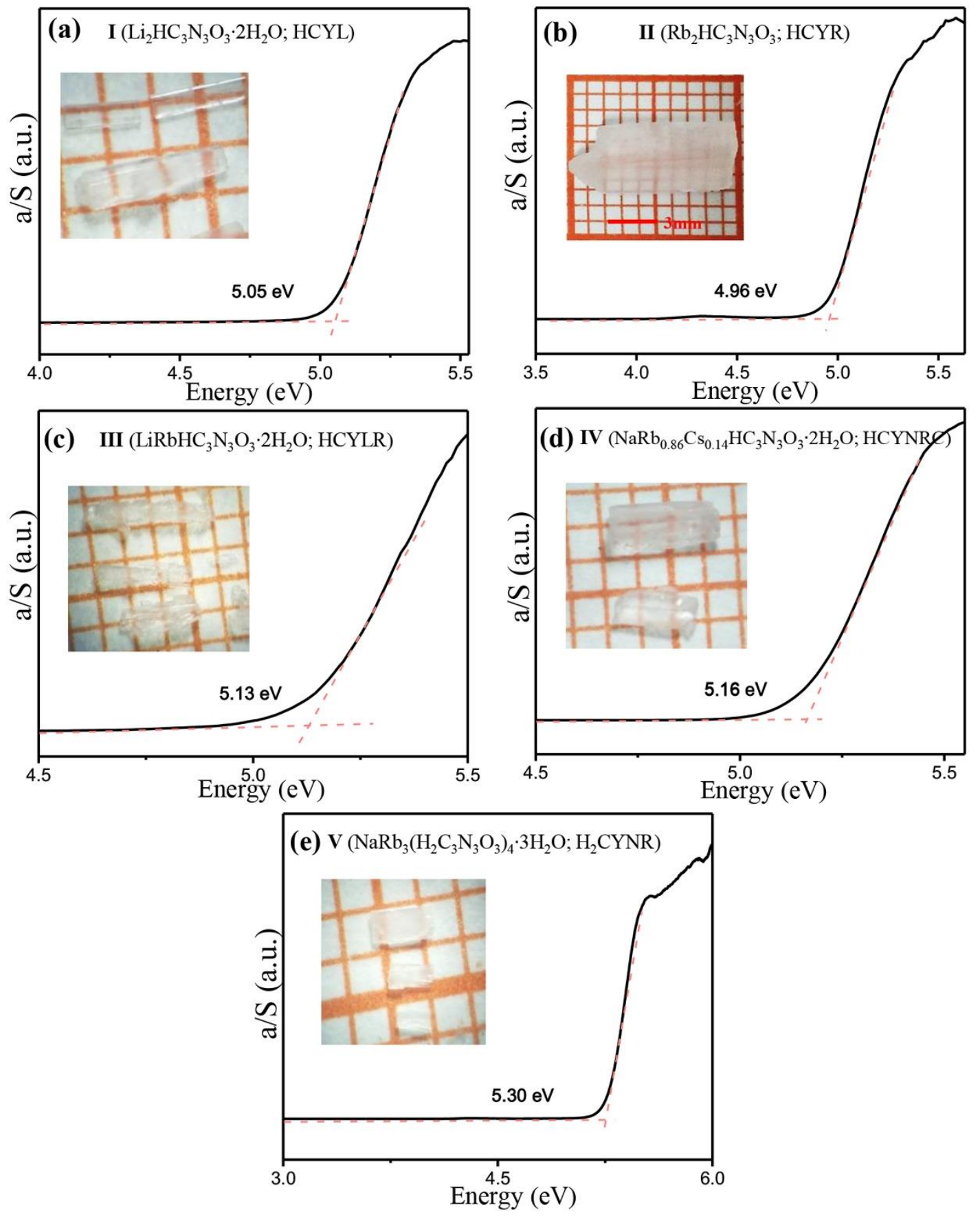

Figure S4. Experimental band gaps of I; HCYL (a), II; HCYR (b), III; HCYLR (c), IV; HCYNRC (d) and $\mathbf{V} ; \mathrm{H}_{2}$ CYNR (e). The inset of patterns shows the colorless crystals of $\mathbf{I}-\mathbf{V}$. 

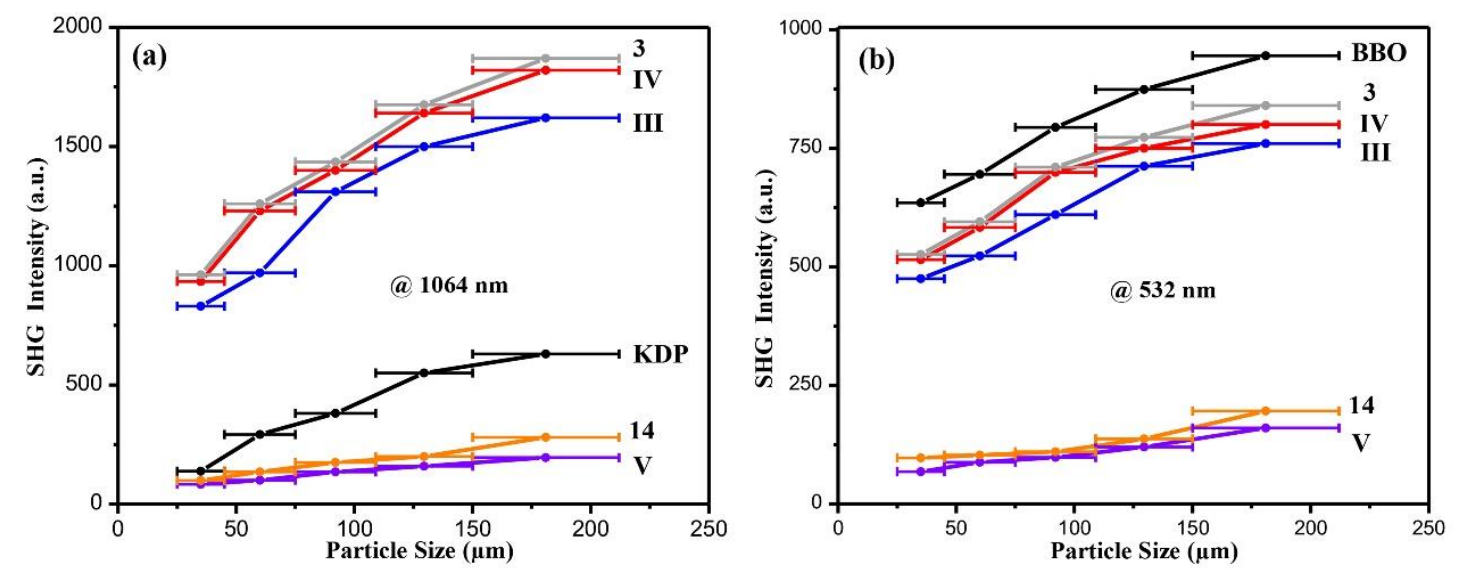

Figure S5. Powder SHG measurements of III; HCYLR, IV; HCYNRC, V; $\mathrm{H}_{2}$ CYNR and two synthesized representative compounds, i.e, $\mathbf{3}$ (HCYLK) ${ }^{16}$ and $\mathbf{1 4}\left(\mathrm{H}_{2} \mathrm{CYNC}\right)^{18}$, the SHG intens ities and phase-matchability of 3 and 14 agree well with the refs within the experimental error range, and shows the good reliability and reproducibility of our measurements. Commercial KDP and BBO (bought from Core Optronics Co., Ltd,.) were used as references at $1064 \mathrm{~nm}$ (a) and $532 \mathrm{~nm}$ (b), respectively. 

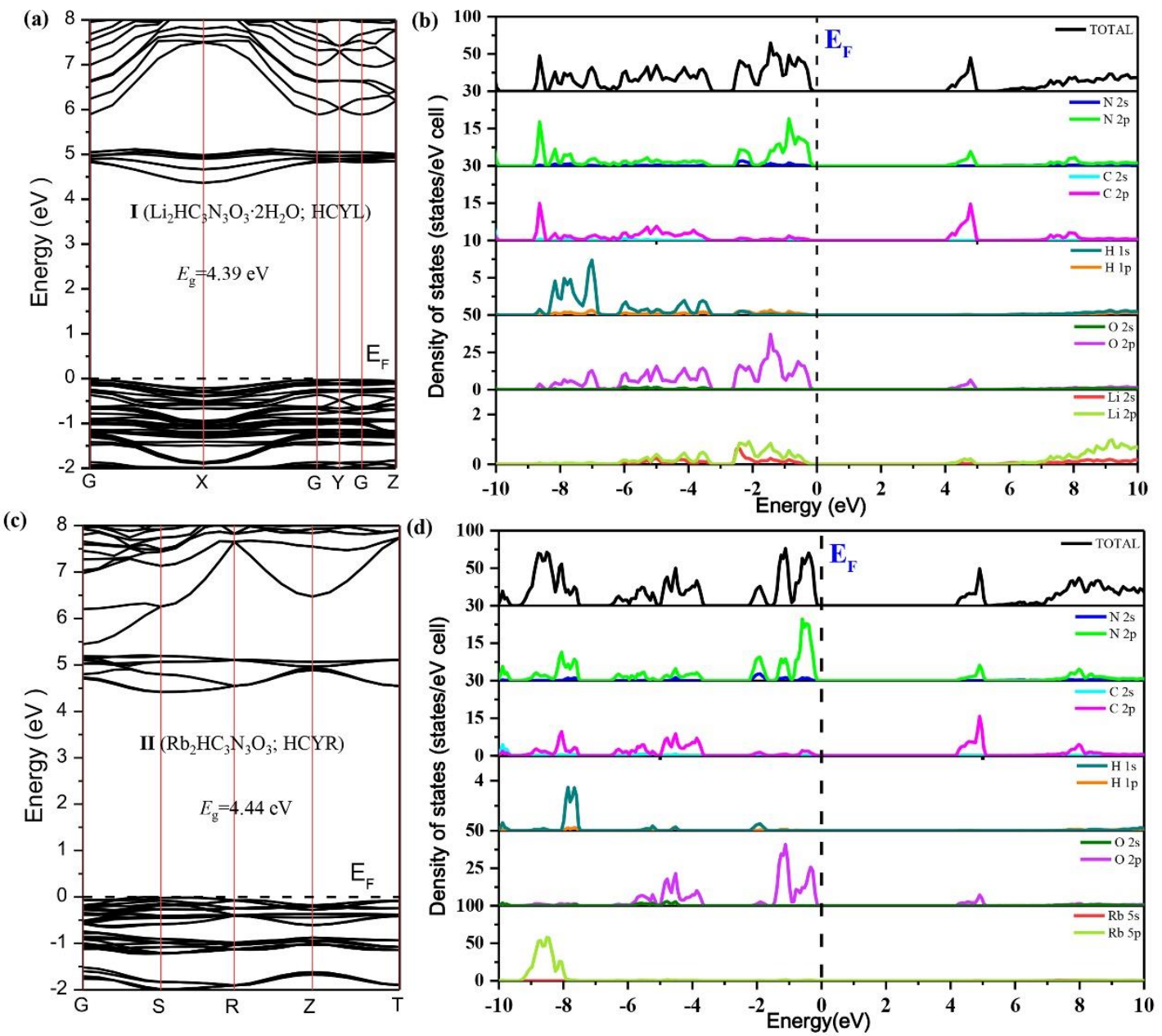

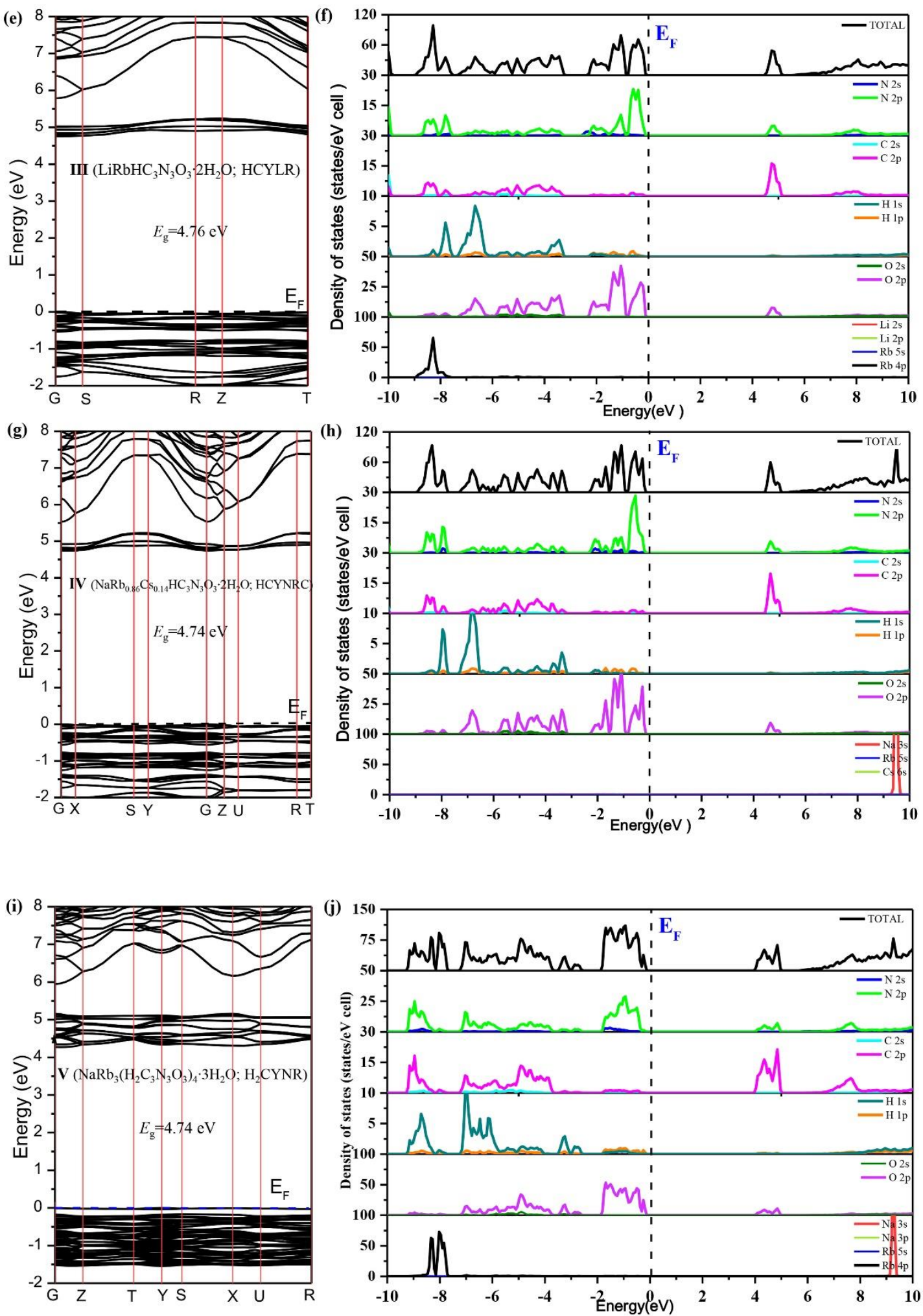

Figure S6. Band structures and density of states for I; HCYL (a, b), II; HCYR (c, d), III; HCYLR (e, f), IV; HCYNRC (g, h) and V; $\mathrm{H}_{2}$ CYNR (i, j). 


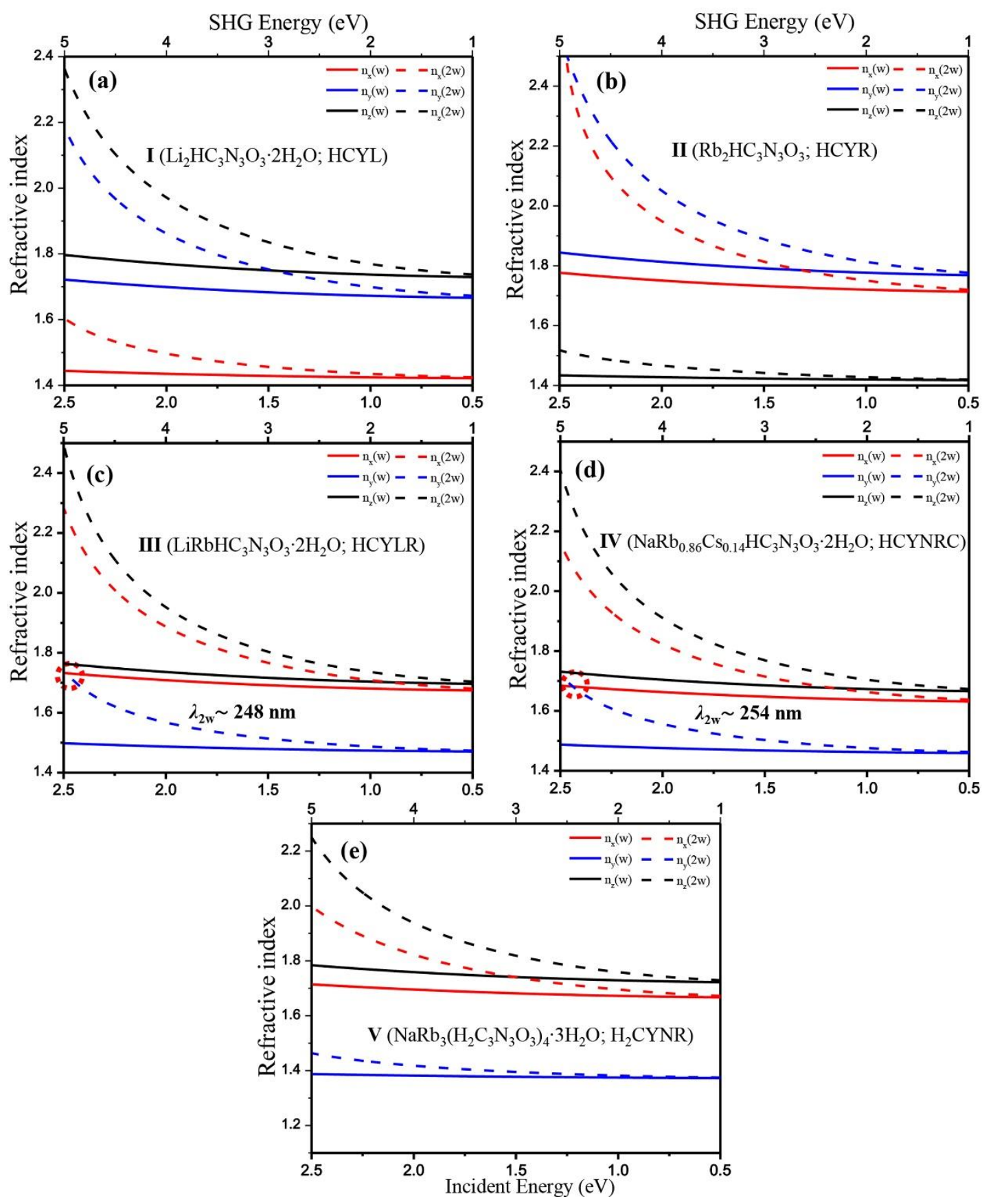

Figure S7. The calculated refractive index dispersion curves of I; HCYL (a), II; HCYR (b), III; HCYLR (c), IV; HCYNRC (d) and V; $\mathrm{H}_{2}$ CYNR (e). (The shortest phase-matched wavelengths are marked as red dotted rings). 

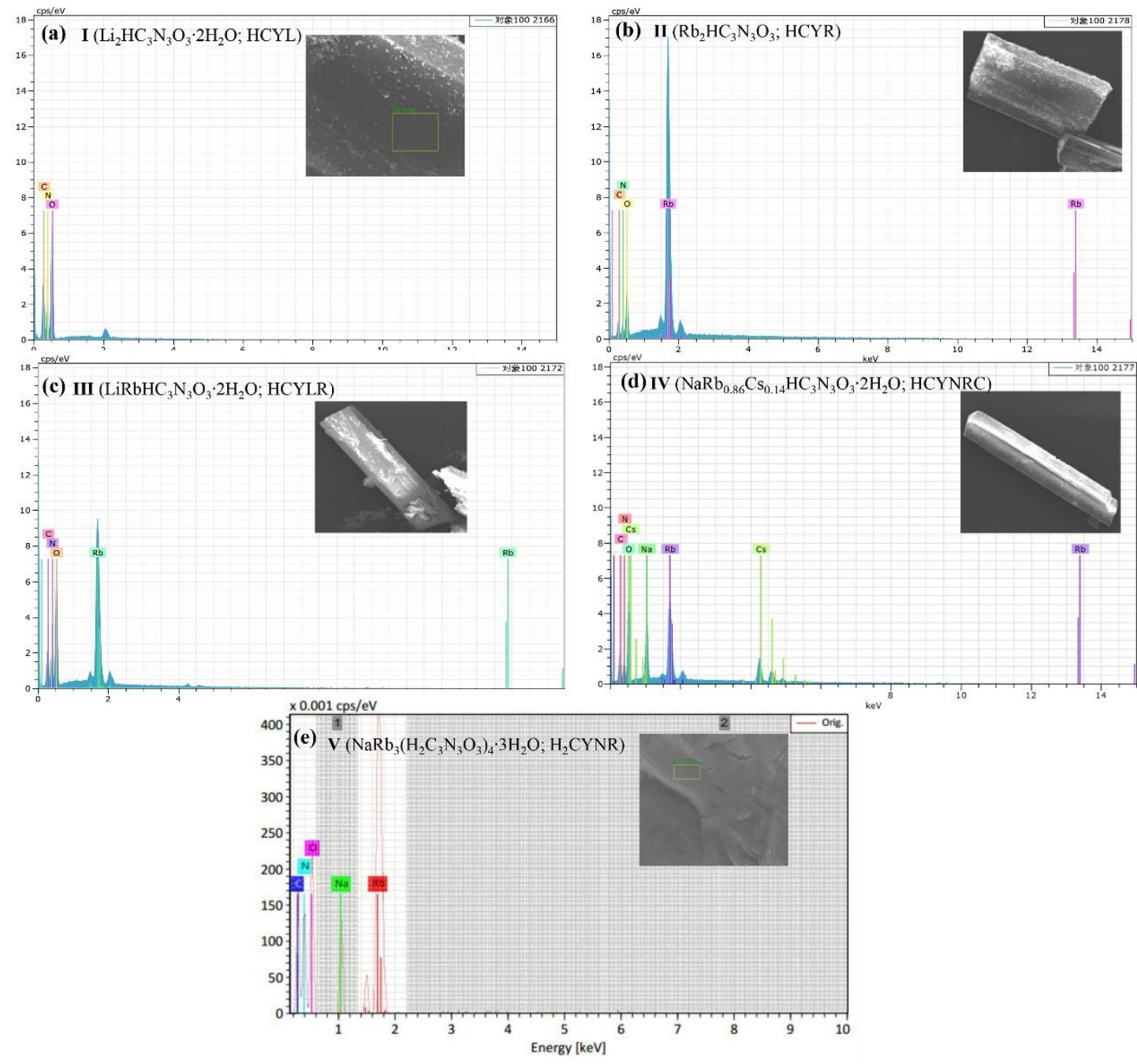

Figure S8. Energy dispersive X-ray spectroscopy (EDS) analysis of I; HCYL (a), II; HCYR (b), III; HCYLR (c), IV; HCYNRC (d) and V; $\mathrm{H}_{2}$ CYNR (e). 

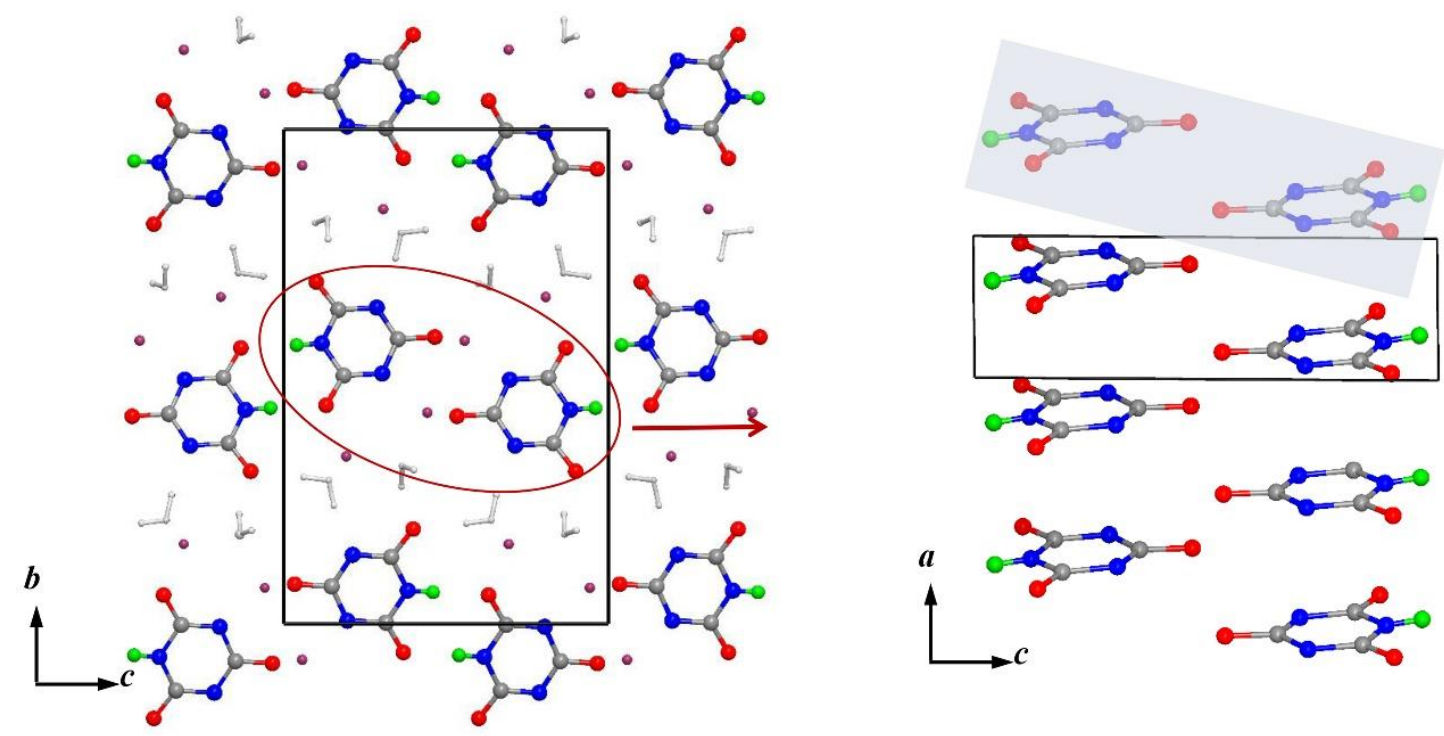

Figure S9. The crystal structure of $\left.\mathbf{I}\left(\mathrm{Li}_{2}\left(\mathrm{HC}_{3} \mathrm{~N}_{3} \mathrm{O}_{3}\right) \cdot 2 \mathrm{H}_{2} \mathrm{O} ; \mathrm{HCYL}\right)\right)$. The right pattern shows the stacking of the $\mathrm{HCY}$ rings along $a$ direction (hereafter: red, O; dark grey, $\mathrm{C}$; blue, N; green, $\mathrm{H}$ and purple, Li; light grey, $\mathrm{H}_{2} \mathrm{O}$ molecules).

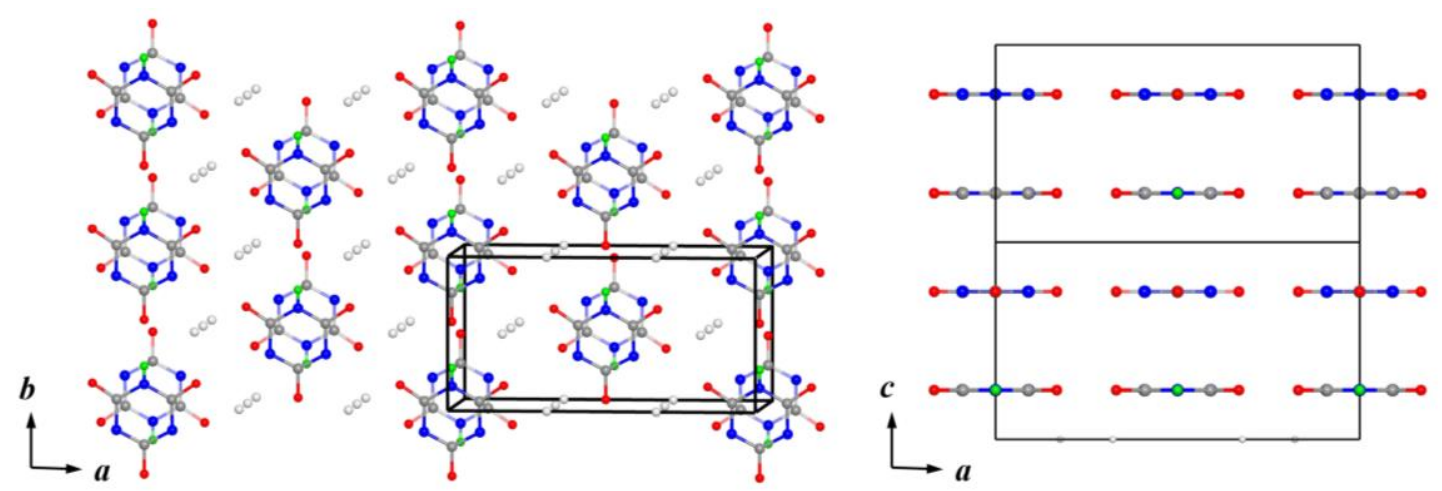

Figure S10. The crystal structure of II $\left.\left(\mathrm{Rb}_{2}\left(\mathrm{HC}_{3} \mathrm{~N}_{3} \mathrm{O}_{3}\right) ; \mathrm{HCYR}\right)\right)$, the right pattern shows the stacking of the HCY rings along $c$ axis in II. (light grey, $\mathrm{Rb}$ ). 


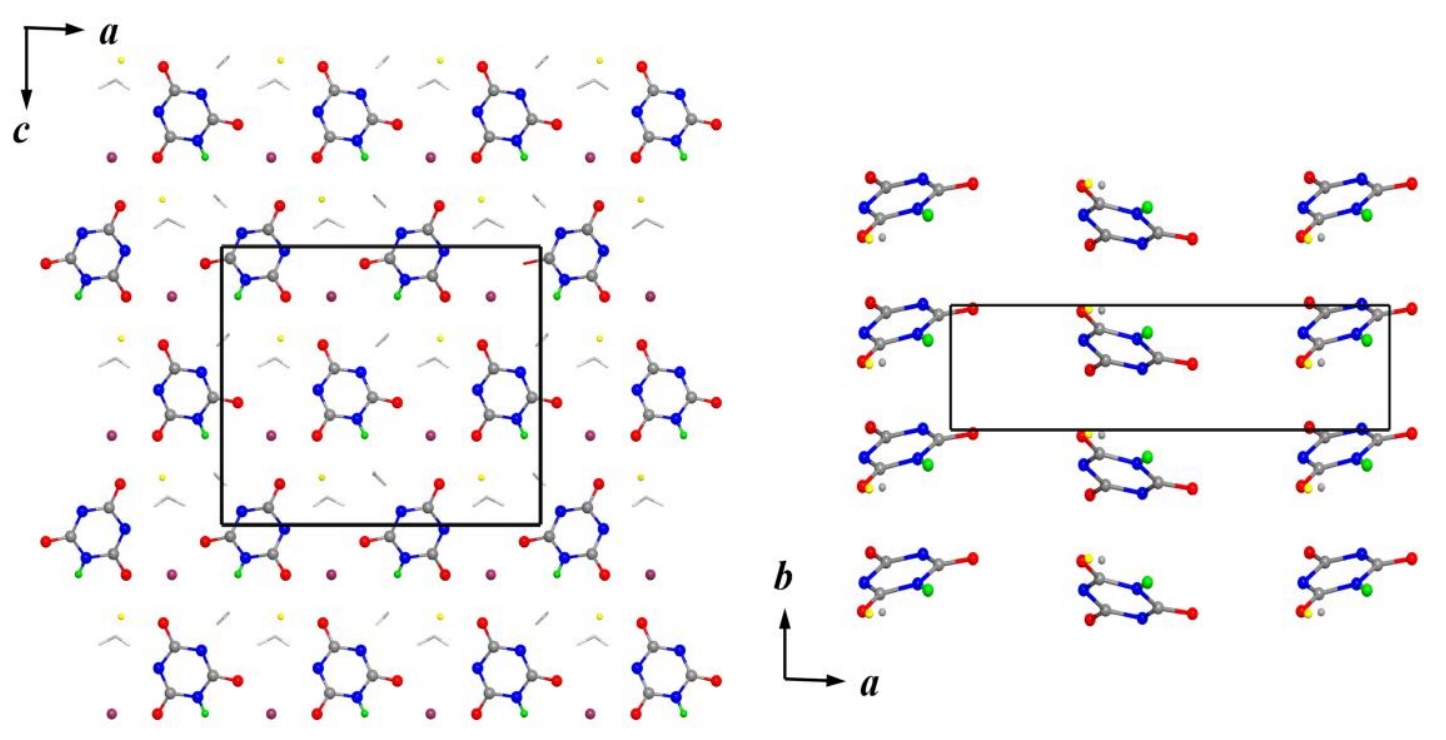

Figure S11. The crystal structure of III $\left(\mathrm{LiRb}\left(\mathrm{HC}_{3} \mathrm{~N}_{3} \mathrm{O}_{3}\right) \cdot 2 \mathrm{H}_{2} \mathrm{O} ; \mathrm{HCYLR}\right)$ or IV $\left(\mathrm{NaRb}_{0.86} \mathrm{Cs}_{0.14}\left(\mathrm{HC}_{3} \mathrm{~N}_{3} \mathrm{O}_{3}\right) \cdot 2 \mathrm{H}_{2} \mathrm{O} ; \mathrm{HCYNRC}\right)$, the right pattern shows the stacking of the HCY rings along $b$ axis in III or IV (For clarity, half of the CY rings are not drawn).
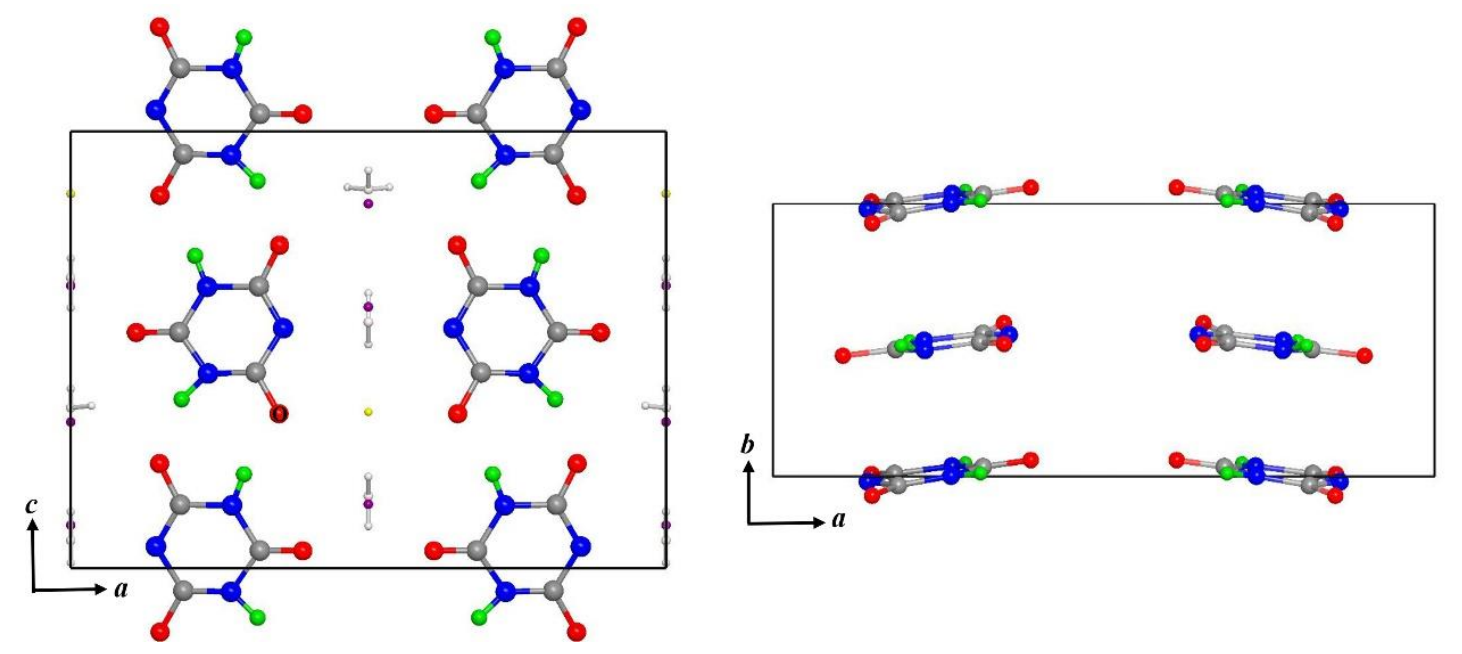

Figure S12. The crystal structure of $\mathbf{V}\left(\mathrm{NaRb}_{3}\left(\mathrm{H}_{2} \mathrm{C}_{3} \mathrm{~N}_{3} \mathrm{O}_{3}\right)_{4} \cdot 3 \mathrm{H}_{2} \mathrm{O} ; \mathrm{H}_{2} \mathrm{CYNR}\right)$, the right pattern shows the stacking of the $\mathrm{H}_{2} \mathrm{CY}$ rings along $b$ axis. 

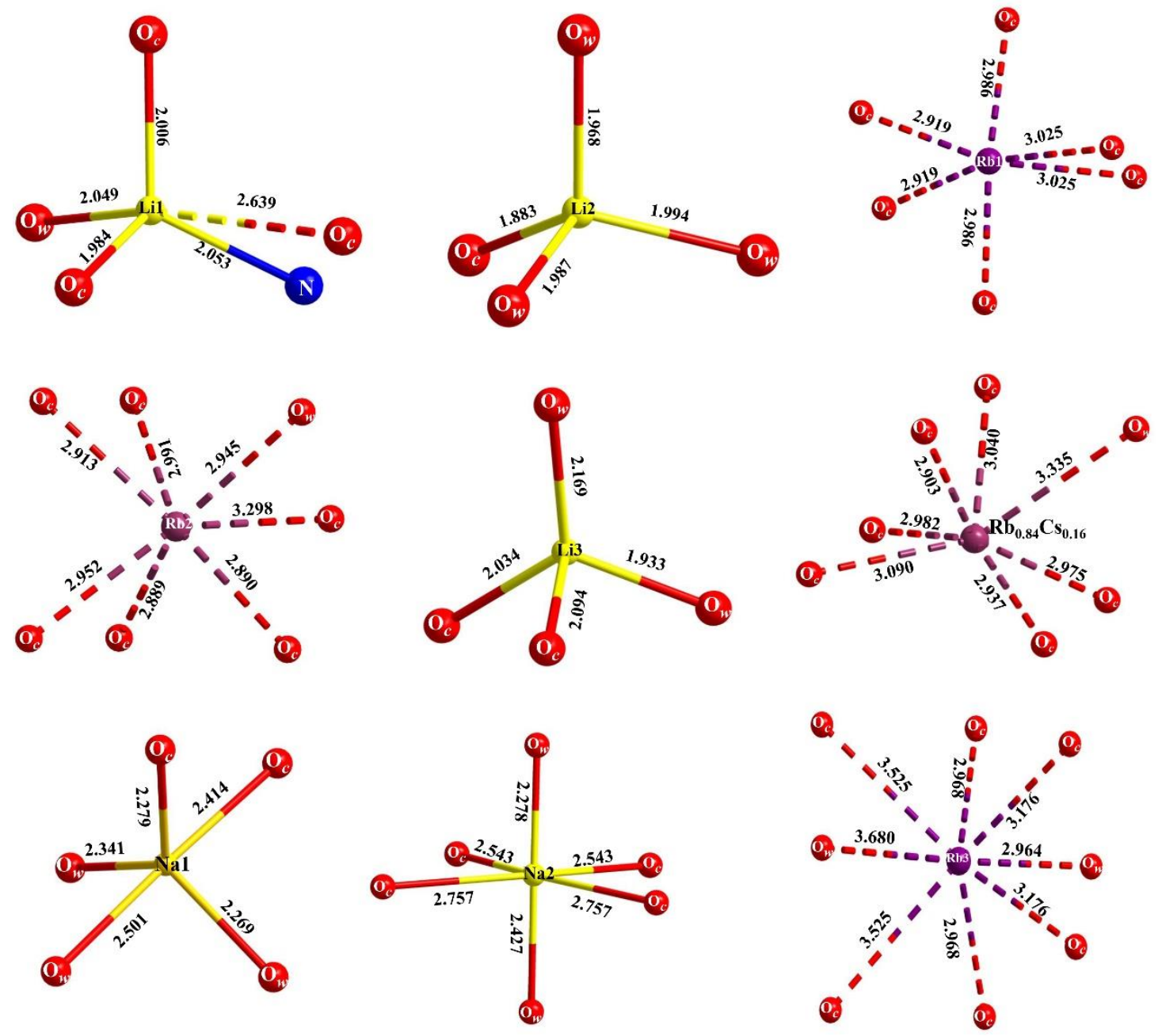

Figure S13. Coordination environment of cations in I; HCYL (Li1 and Li2), II; HCYR (Rb1), III; HCYLR (Rb2 and Li3), IV; HCYNRC ( $\mathrm{Rb}_{0.84} \mathrm{Cs}_{0.16}$ and Na1) and $\mathrm{H}_{2} \mathrm{CYNR}(\mathrm{Na} 2$ and $\mathrm{Rb} 3$ ). 

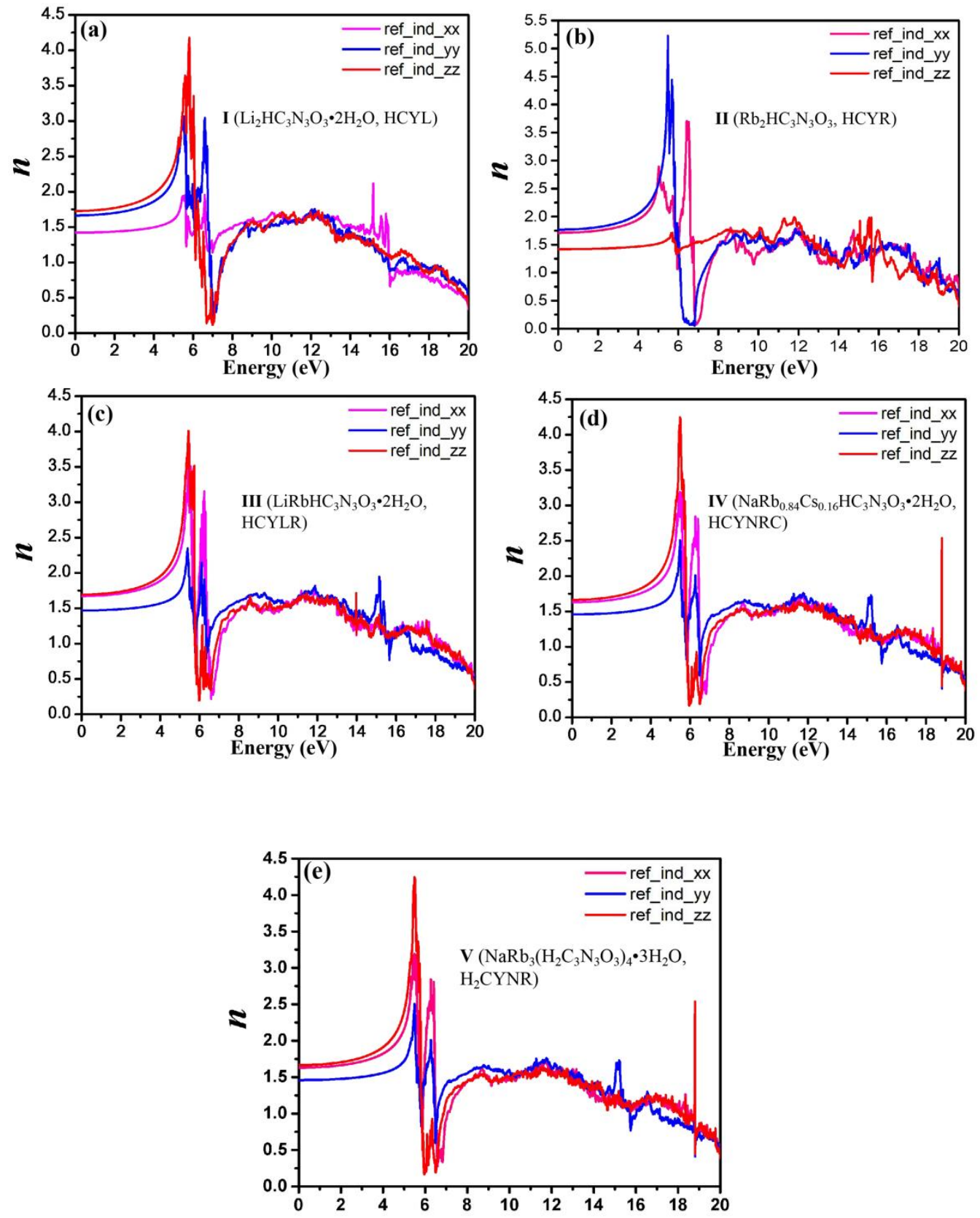

Figure S14. The calculated refractive index ( $n$ ) variations of I; HCYL (a), II; HCYR (b), III; HCYLR (c), IV; HCYNRC (d) and V; $\mathrm{H}_{2}$ CYNR (e). 

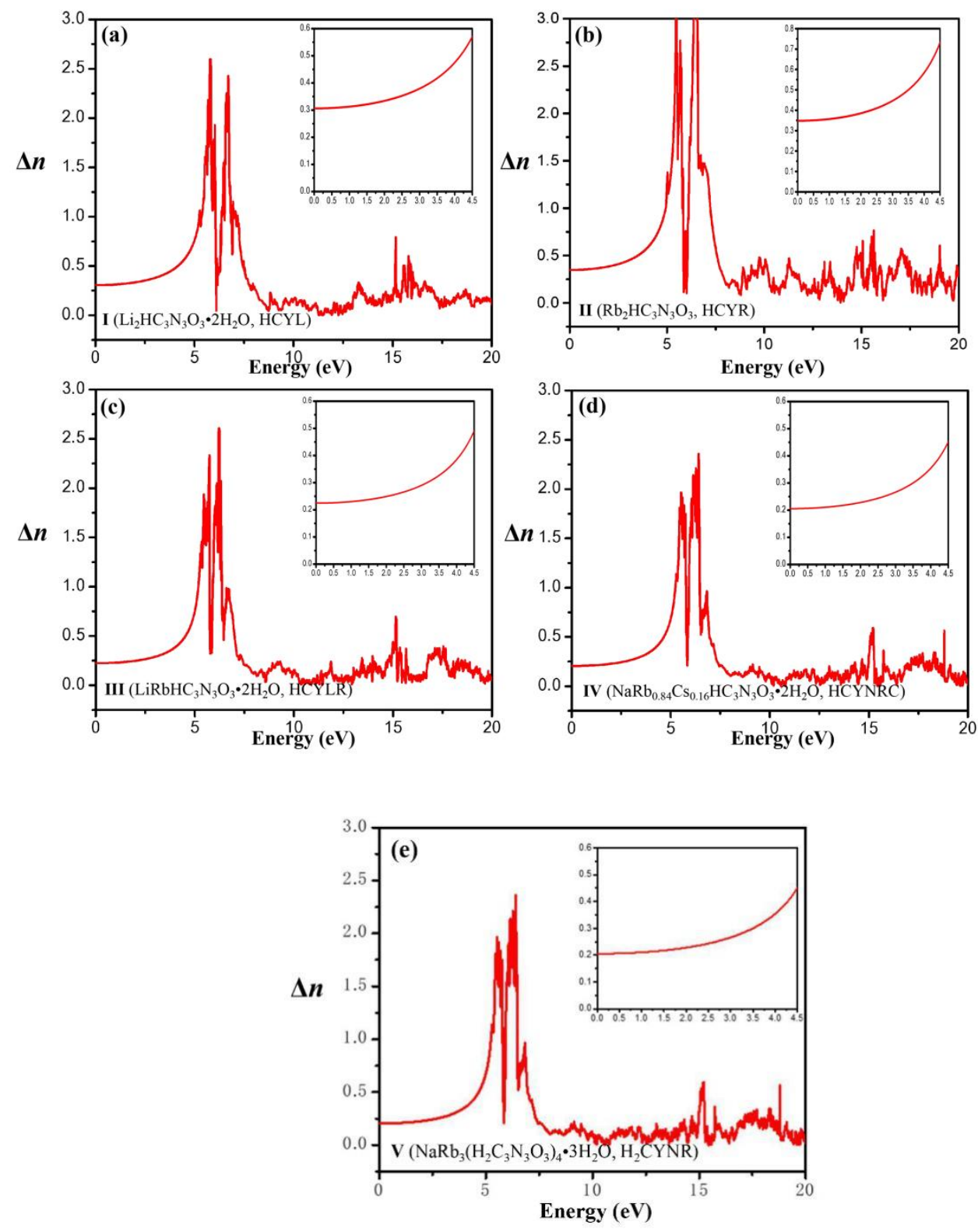

Figure S15. Calculated birefringence $(\Delta n)$ of I; HCYL (a), II; HCYR (b), III; HCYLR (c), IV; HCYNRC (d) and V; $\mathrm{H}_{2}$ CYNR (e) 


\section{References}

(S1) Gross, P.; Höppe, H. A. The Sodium (iso) cyanurates $\mathrm{Na}_{\mathrm{x}}\left[\mathrm{H}_{3}-\mathrm{xC}_{3} \mathrm{~N}_{3} \mathrm{O}_{3}\right] \cdot \mathrm{yH}_{2} \mathrm{O}(\mathrm{x}=1-3, \mathrm{y}=0,1)$ : A key-series for understanding the crystal chemistry of metal (iso) cyanurates. ZAnorg Allg Chem. 2019. 645, 257-266;

(S2) Kalmutzki, M.; Ströbele, M.; Wackenhut, F.; Meixner, A. J.; Meyer, H. J. Synthesis, structure, and frequency-doubling effect of calcium cyanurate. Angew. Chem. Int. Edit. 2014, 53, 14260-14263.

(S3) Chen, J.; Xiong, L.; Chen, L. Wu, L. M. $\mathrm{Ba}_{2} \mathrm{NaClP}_{2} \mathrm{O}_{7}$ : unprecedented phase matchability induced by symmetry breaking and its unique Fresnoite-type structure. J. Am. Chem. Soc. 2018, 140, 1408214086.

(S4) Duan, R. H.; Liu, P. F.; Lin, H.; Zheng, Y. J.; Yu, J. S.; Wu, X. T.; Huang-Fu, S. X.; Chen, L. Ba 6 Li ${ }_{2} \mathrm{CdSn}_{4} \mathrm{~S}_{16}$ : lithium substitution simultaneously enhances band gap and SHG intensity. J. Mater. Chem.C. 2017.5, 7067-7074.

(S5) Lin, H.; Chen, L.; Zhou, L. J.; Wu, L. M. Functionalization based on the substitutional flexibility: strong middle IR nonlinear optical selenides $\mathrm{AX}_{4}^{\mathrm{II}} \mathrm{X}_{5}^{\mathrm{III}} \mathrm{Se}_{12}$. J. Am. Chem. Soc. 2013, 135, 12914 12921.

(S6) Kurtz, S. K.; Perry, T. T. A powder technique for the evaluation of nonlinear optical materials. $J$. Appl. Phys. 1968, 39, 3798-3813.

(S7) Xiong, L.; Chen, J.; Lu, J.; Pan, C. Y.; Wu, L. M. Monofluorophosphate: a new source of deep-ultraviolent nonlinear optical materials. Chem. Mater. 2018. 30, 7823-7830.

(S8) Kalmutzki, M.; Strobele, M.; Meyer, H. J. From cyanate to cyanurate: cyclotrimerization reactions towards the novel family of metal cyanurates. Dalton Trans. 2013, 42, 12934-12939

(S9) Li, Z.; Liang, F.; Guo, Y. W.; Lin, Z. S.; Yao, J. Y.; Zhang, G. C.; Chen, C. T. $\mathrm{Ba}_{2} \mathrm{M}\left(\mathrm{C}_{3} \mathrm{~N}_{3} \mathrm{O}_{3}\right)_{2}(\mathrm{M}=$ $\mathrm{Mg}, \mathrm{Ca}$ ): potential UV birefringent materials with strengthened optical anisotropy originating from the $\left(\mathrm{C}_{3} \mathrm{~N}_{3} \mathrm{O}_{3}\right)^{3-}$ group. J. Mater. Chem. 2018. 6, 12879-12887.

(S10) Sch mid, S.; Schnick, W. Synthese und kristallstruktur des ersten oxonitridoborates $-\mathrm{Sr}_{3}\left[\mathrm{~B}_{3} \mathrm{O}_{3} \mathrm{~N}_{3}\right]$. Z. Anorg. Allg. Chem. 2002, 628, 1192-1195

(S11) Tang, J.; Liang, F. Meng, X. H.; Kang, K. J.; Yin, W. L.; Zeng, T. X.; Kang, B.; Xia, M. J.; Lin, Z. S.; Yao, J. Y.; Zhang, G. C.; Kang, B. $\mathrm{Ba}_{3}\left(\mathrm{C}_{3} \mathrm{~N}_{3} \mathrm{O}_{3}\right)_{2}$ : A new phase of barium cyanurate containing parallel $\pi$-conjugated groups as a birefringent material replacement for calcite. Cryst Growth Des. 2018. $19,568-572$. 